\title{
Dendritic signal integration in a Drosophila Mushroom Body Output Neuron (MBON) essential for learning and memory
}

\author{
Omar A. Hafez *† \\ Benjamin Escribano $* \ddagger$ \\ Rouven L. Ziegler ${ }^{\ddagger}$ \\ Jan J. Hirtz ${ }^{\S}$ \\ Ernst Niebur ${ }^{\dagger}$ \\ Jan Pielage $\mathrm{e}^{\ddagger}$
}

March 4, 2021

\begin{abstract}
The ability to associate neutral stimuli with either positive or negative valence forms the basis for most forms of decision making. Long-term memory formation then enables manifestation of these associations to guide behavioral responses over prolonged periods of time. Despite recent advances in the understanding of the neuronal circuits and cellular mechanisms controlling memory formation, the computational principles at the level of individual information processing modules remain largely unknown. Here we use the Drosophila mushroom body (MB), the learning and memory center of the fly, as a model system to elucidate the cellular basis of memory computation. Recent studies resolved the precise synaptic connectome of the MB and identified the synaptic connections between Kenyon cells (KCs) and mushroom body output neurons (MBONs) as the sites of sensory association. We build a realistic computational model of the $\mathrm{MBON}-\alpha 3$ neuron including precise synaptic connectivity from the 948 upstream KCs innervating the $\alpha \beta$ MB lobes. To model membrane properties reflecting in vivo parameters we performed patch-clamp recordings of $\mathrm{MBON}-\alpha 3$. Based on the in vivo data we model synaptic input of identified individual cholinergic $\mathrm{KC}>\mathrm{Mbon}$ synapses by local conductance changes at the dendritic sections defined by the electron microscopic reconstruction. Modelling of activation of all individual synapses confirms prior results demonstrating that this neuron is electrotonically compact. As a likely consequence, activation pattern of individual KCs with identical numbers of synaptic connection but innervating different sections of the MBON- $\alpha 3$ dendritic tree result in highly similar depolarization voltages at its soma and likely spike initiation zone. Furthermore, we show that KC input patterns reflecting physiological activation by individual odors in vivo are sufficient to robustly drive MBON spiking. Our data suggest that the sparse innervation by KCs can efficiently control or modulate MBON activity, with minimal requirements on the specificity of synaptic localization. The KC>Mbon architecture therefore provides a suitable module to incorporate different olfactory associative memories based on stochastically encoded odorspecificity of KCs.
\end{abstract}

\section{Introduction}

Navigating within a complex environment requires organisms to discriminate between a vast amount of potential rewarding and stressful interactions. Learning to avoid potentially harmful interactions will promote survival of individual animals and is manifested by long term memory (LTM) mechanisms. In recent years, significant progress has been made towards our understanding of the cellular and circuit mechanisms underlying learning and memory. Despite these advances, at the computational level it remains largely unknown how cellular and circuit architecture contributes to the efficient formation and storage of multiple parallel memories.

Here, we use the Drosophila melanogaster mushroom body (MB) as a simple model system to investigate the computational principles underlying learning and memory. A key advantage of the MB, the learning and memory center of the fly, is its relative simplicity in terms of number and types of

\footnotetext{
*Equal contribution

${ }^{\dagger}$ Zanvyl Krieger Mind/Brain Institute, Johns Hopkins University, Baltimore, MD 21218, USA

$\ddagger$ Division of Neurobiology and Zoology, Department of Biology, University of Kaiserslautern, D-67663 Kaiserslautern, Germany

$\S$ Physiology of Neuronal Networks Group, Department of Biology, University of Kaiserslautern, D-67663 Kaiserslautern, Germany

ISolomon Snyder Department of Neuroscience, Johns Hopkins University, Baltimore, MD 21218, USA

$\|$ To whom correspondence should be addressed: pielage@bio.uni-kl.de
} 
neurons compared to the mammalian brain. Numerous studies demonstrated that the MB is required for the acquisition and recall of associative olfactory memories $[22,43,51,53,63,88]$ and a wide variety of genetic tools are available to access and manipulate key cell types within the memory circuitry $[23,25,42,44,45,50,60,67,79,84]$. The formation of olfactory memory within the MB circuit depends on the association of normally neutral odors with either positive (reward learning) or negative (avoidance learning) valences [49]. Odor information is transmitted from the antennal lobe via projection neurons (PNs) to the Kenyon cells (KCs) of the MB [89, 90]. PNs are connected in a largely stochastic manner to the approximately $2000 \mathrm{KCs}$ per brain hemisphere resulting in a unique $\mathrm{KC}$ odor representation in all individual flies $[3,15,30,47,48,83,91]$.

Calcium imaging studies demonstrated that any given odor activates approximately $5 \%$ of KCs [14, $39,75,87]$. KCs then transmit this odor information to mushroom body output neurons (MBONs) that subdivide the MB lobes into distinct structural and functional modules by virtue of their dendritic arborizations $[19,33,34,59,61,64,86]$. During associative olfactory conditioning the behavioral response to any given odor can be altered to encode positive (approach) or negative (avoidance) behavior. The valence of the conditioned response is encoded by the activation of two classes of dopaminergic neurons (DANs) that selectively innervate the structural MB modules defined by the MBON dendrites [3]. DANs of the PPL1 cluster preferentially provide input to the vertical lobe of the MB and are activated during negative reinforcement while DANs of the PAM cluster are activated during positive reinforcement and modulate activity in the horizontal lobe of the $\mathrm{MB}[4,7,12,20,33,40,49,68,74,85,86]$. The majority of MBONs of the vertical lobe utilize acetylcholine as a neurotransmitter and artificial activation of these MBONs often mediates approach behavior [5]. In contrast, the majority of MBONs of the horizontal lobe releases glutamate as a neurotransmitter and mediates avoidance behavior [5]. In vivo calcium imaging and electrophysiological experiments demonstrated that the activity of individual MBONs can be altered by conditioning paradigms $[24,34,41,59,62,64,66]$. This modulation of MBON activity depends on pairing of DAN and KC activity and results in either an enhancement or depression of the strength of the $\mathrm{KC}>$ Mbon connection [4, 12, 20, 33, 40, 59]. These changes in KC>Mbon connection strength are incorporated in either the horizontal or the vertical lobe depending on the administered stimulus. As a consequence, the resulting net activity of all MBONs is shifted to either approach or avoidance behavior and the behavioral response towards the conditioned stimulus during memory recall is changed [4, 12, 20, 33, 40, 59]. Evidence for this MBON balance model [19] has been provided for short term memory $[5,21,34,61,64]$ but how such a model is incorporated during long term memory still remains largely unclear. In addition, it is not yet resolved whether all MBONs contribute to the simple balance model, and to what extent more complicated network modules contribute to the computation of memory-dependent behavioral output $[3,5,5,41,62,66]$. Furthermore, studies have demonstrated that protein synthesis in MBONs is also required for the formation and recall of memories [62, 93], indicating that synaptic plasticity may not be restricted to the presynaptic KC input.

Recently, genetic tools have been created that enable genetic tagging of long-term memory engram cells in the MB $[54,76]$. These studies indicate that modulation of a relatively small number of KCs is sufficient to significantly alter memory recall. To determine the impact of populations of KCs on MBON activity and to resolve potential computational mechanisms underlying memory encoding we utilize the recently published synaptic connectome of the Drosophila MB as a template [81, 95] that precisely defined the input patterns of all KCs innervating specific MBONs. Here we focus on MBON- $\alpha 3$ (MBON 14), an MBON at the tip of the $\alpha$ lobe that is relevant for LTM $[5,66]$. We first determine the physiological parameters of MBON- $\alpha 3$ by patch clamp electrophysiology. We then use these parameters to generate an anatomically and physiologically realistic in silico model of $\mathrm{MBON}-\alpha 3$ and its $\mathrm{KC}$ inputs. Using a variety of computational simulations we provide evidence that MBON- $\alpha 3$ is electrotonically compact and perfectly suited to incorporate a substantial number of parallel memories based on largely stochastic KC input.

\section{Results}

\subsection{Electrophysiological characterisation of $\mathrm{MBON}-\alpha 3$}

MBON- $\alpha 3$ is particularly suitable for computational modeling due to the availability of its complete dendritic reconstruction through electron microscopy [81]. In addition, we can easily access MBON$\alpha 3$ genetically by using a specific Gal4 line (MB082C-Gal4) to label MBON- $\alpha 3$ by the expression of membrane-bound 10XUAS-IVS-mCD8::GFP (Figure 1 (A)). In immunohistochemical co-labellings with the active zone marker Bruchpilot (Brp) we were able to visualize the dendritic input sites, the axonal projections and the cell soma of $\mathrm{MBON}-\alpha 3$ (Figure 1 (A), arrow). We used this labelling to perform 
fluorescence-guided electrophysiological patch-clamp whole-cell recordings of this neuron ex vivo (Figure $1(\mathrm{C}) \&(\mathrm{D}))$. We carried out standard patch-clamp protocols in five independent preparations and recorded all essential physiological parameters (see Table 1). First, we performed a continuous currentclamp protocol over $60 \mathrm{~s}$ at a sampling rate of $50 \mathrm{kHz}$ which allowed us to determine the average resting membrane potential as $-56.7 \pm 2.0 \mathrm{mV}$. This value for the resting membrane potential is in line with prior measurements of MBONs in vivo [34]. In these recordings we observed spontaneous firing activity of MBON- $\alpha 3$ with an average frequency of $12.1 \mathrm{~Hz}$.

Next, we performed a current-clamp de- and hyper-polarization protocol where we recorded $400 \mathrm{~ms}$ sweeps at a sampling rate of $50 \mathrm{kHz}$. Each sweep started with $0 p A$ for $10 \mathrm{~ms}$, followed by a current injection of $-26 p A$ to $32 p A$, with $2 p A$ increments for $400 \mathrm{~ms}$. These protocols allowed us to evoke action potentials and to determine resulting changes in membrane potential. An example displaying selected traces of a representative recording is shown in Figure $1(\mathrm{~F})$. To determine the membrane time constant we recorded 35 sweeps per cell in a current-clamp injection protocol with injections of $10 p A$ current pulses for $100 \mathrm{~ms}$ at a sampling rate of $20 \mathrm{kHz}$. We averaged 105 traces from 3 cells to determine $\tau_{m}$ as $15.6 \pm 2.3 \mathrm{~ms}$ (Figure $1(\mathrm{G})$; see Figure S1 for raw trace data). We then performed a voltage-clamp step protocol without compensating for the series resistance. In this experiment we recorded at $20 \mathrm{kHz}$ while applying a $5 \mathrm{mV}$ voltage step for $100 \mathrm{~ms}$. This allowed us to calculate the membrane resistance as $R_{m}=926 \pm 55 M \Omega$, a rather high membrane resistance when compared to similarly sized neurons[28]. The neuron is thus highly excitable, with small input currents being sufficient to significantly alter its membrane potential $[28,71]$. The amplitude of action potentials was rather small and similar to previously reported values for MBONs [34]. These small amplitudes are likely a result of the unipolar morphology of the neuron in which signal propagation may bypass the soma (Figure 2 (A) [28]). We measured the neuron's membrane capacitance as $C_{m}=16.76 \pm 1.90 p F$, which classifies this cell as a mid-sized neuron $[28,29,31]$.

To validate these results, we performed multiple analyses of three example cells using the currentclamp de- and hyper-polarization protocols (Figure $2(\mathrm{~A})-(\mathrm{C})$ ). We recorded the absolute deflection, the action potential firing frequency and the amplitude of action potentials for all individual cells. In all three cells we observed that the absolute deflection of the membrane potential was proportional to the increasing current injections (Figure 2 (D)). The action potential firing frequency increased gradually with increasing membrane potential deflections (Figure $2(\mathrm{E})$ ), with no significant change in action potential amplitudes (Figure $2(\mathrm{~F})$ ). This is consistent with the general idea that action potentials are "all or nothing" events, and that MBON- $\alpha 3$ is a spike-frequency adapting neuron. These values and the tunability of the MBON$\alpha 3$ are in good agreement with prior recordings of a different class of MBONs and of other central neurons in Drosophila [35]. We next used these data to generate an in silico model based on realistic passive membrane properties.

\subsection{Constructing an in silico model of $\operatorname{MBON}-\alpha 3$}

For our in silico model of MBON- $\alpha 3$ we used the recently published electron microscopy based connectome of the Drosophila mushroom body [81, 95]. This dataset includes the precise morphological parameters of the entire dendritic tree, the axon terminals and parts of the neurite connection to the cell soma. This dataset also included precise location data for all 12,770 synaptic connections from the 948 innervating KCs. To build a complete morphological reconstruction of MBON- $\alpha 3$ we determined the length of the soma to dendrite neurite via confocal light microscopy and we incorporated this information together with all other values into the NEURON simulation environment [38] (Figure 3 (A) \& (C); see section 4.4 and Table S2 and Figure S3 for morphological details). Based on the published vector data [80, 95] we subdivided the dendritic tree into 4,336 linear sections, of which 3,121 are postsynaptic to one or more of the 12,770 synaptic contacts from $948 \mathrm{KCs}$ (Figure $3 \mathrm{~B}$ ). We used linear cable theory [55, 56] to generate the in silico model of $\mathrm{MBON}-\alpha 3$. Linear cable theory is the baseline model for neurites and the most rational approach in the absence of detailed information about potential nonlinear currents.

In previous simulations of Drosophila neurons, the kinetics of membrane polarisation as a response of current injections were adjusted to in vivo measurements through a fitting procedure [28]. We first defined the boundaries of the passive membrane parameters within NEURON's PRAXIS (principle axis) optimization algorithm and incorporated the experimentally defined passive membrane properties (Table 1). We then determined the biophysical parameters of the neuron by current (step) injections of $-10 p A$ for $200 \mathrm{~ms}$ at the soma and recording the neuronal voltage traces. This enabled us to fit the membrane parameters of our model neuron to the experimental data, Figure 3D. The biophysical parameters that resulted from the fitting procedure are provided in the supplement (S3). We obtained membrane kinetics that closely resembles in vivo current injections of $-10 p A$, with a mean squared error between model and measured data of $0.0481 \mathrm{mV}^{2}$. For comparison we normalized both the modelled current injection 

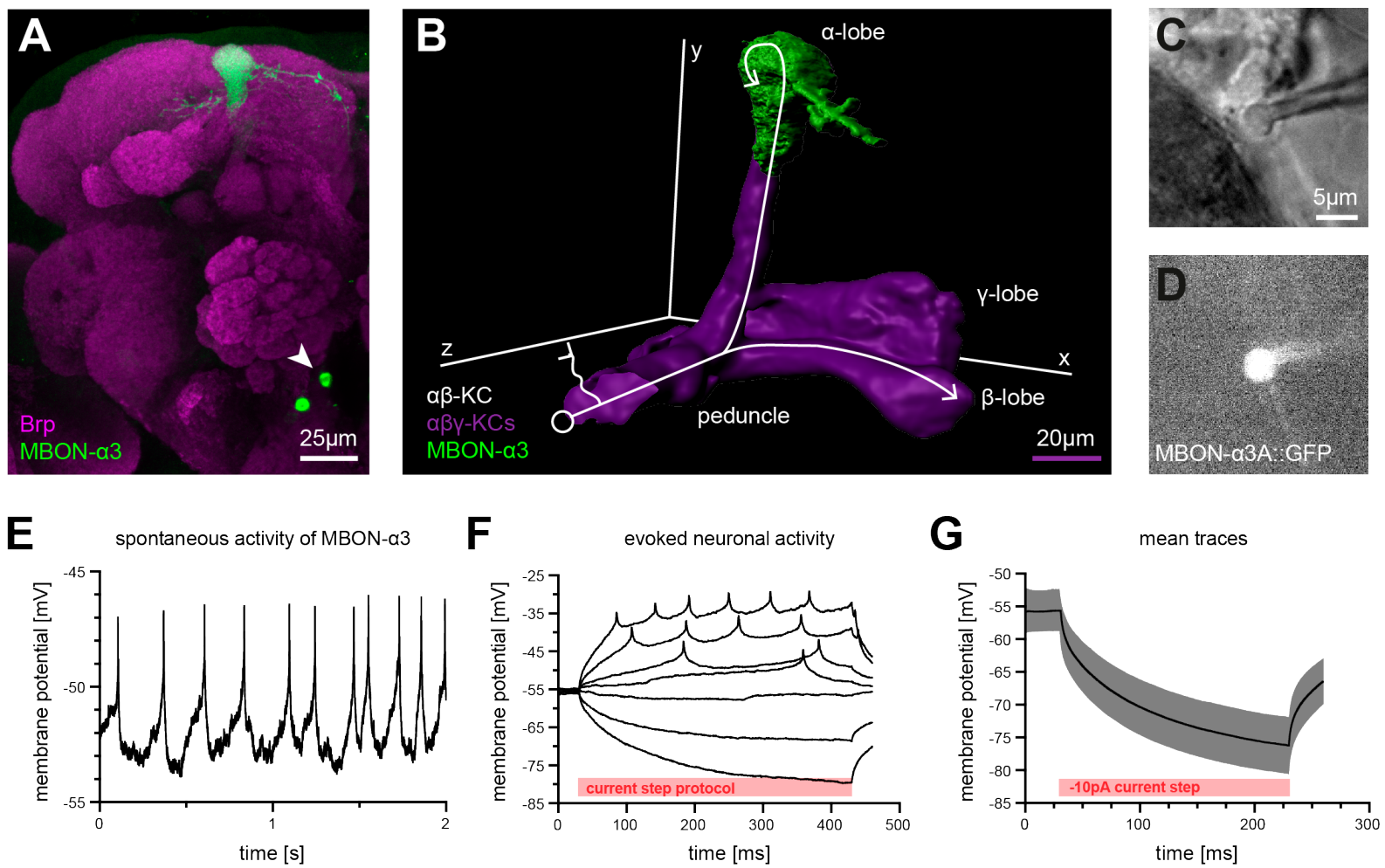

Figure 1 Electrophysiological properties of MBON- $\alpha 3$

(A) Immuno- histological staining of MBON- $\alpha 3$ (green) and bruchpilot (Brp) as a marker for neuronal active zones (magenta). (B) Superimposed renderings of MBON- $\alpha 3$ (green) and $\alpha, \beta, y$ KCs to illustrate KC-MBON connectivity. $A \alpha \beta-K C$ is illustrated in white. (C) MBON- $\alpha 3$ soma attached to a patch pipette. The pipette was guided through light microscopy (C) and fluorescence light microscopy (D). (E) An example of the measured spontaneous activity of $\mathrm{MBON}-\alpha 3$ through patch clamp in the current clamp mode with no current injection. (F) Example for elicited neuronal activity of MBON- $\alpha 3$ through pulsed current injections for $400 \mathrm{~ms}$. From bottom up the trace corresponds to a $-10,-6,-2,0,2,6,10 \mathrm{pA}$ injection. (G) Mean of the elicited neuronal activity through a $200 \mathrm{~ms}$ current injection of -10 pA from 105 traces in 3 different cells.

Figure 1: Electrophysiology of MBON- $\alpha 3$. (A) Immunohistological staining of MBON- $\alpha 3$ (green) and Bruchpilot (Brp) as a marker for synaptic active zones (magenta). The arrowhead marks the cell body of MBON- $\alpha 3$ that is positioned below the antennal lobes and connected via a long neurite to the dendritic and axonal terminal compartments at the dorsal area of the brain. (B) Artificially superimposed partial renderings of MBON- $\alpha 3$ (green) and $\alpha \beta \gamma$-KCs (purple) to illustrate KC-MBON connectivity. A simplified potential $\alpha \beta$-KC innervation is illustrated in white. (C) Transmitted light image of a MBON- $\alpha 3$ soma attached to a patch pipette. (D) Visualisation of the GFP expression of the MBON- $\alpha 3$ that was used to identify the cell via fluorescence microscopy. The patch pipette tip can be seen attached to the soma (right side). Scale bar as in (C). (E) Spontaneous activity of MBON- $\alpha 3$ measured in current clamp mode without current injection. (F) Recording of evoked neuronal activity of MBON- $\alpha 3$ after step-wise increasing current injections of $400 \mathrm{~ms}$ long pulses. Depicted are current pulses in the range of $-10 p A$ (bottom) to $10 p A$ (top), with intermediate values of $-6,-2,0,2,6 p A$. (G) Mean trace of the induced membrane polarization resulting from a $200 \mathrm{~ms}$ long current injection of $-10 \mathrm{p} A$. We combined 105 trials from three different cells (35 each) to determine the membrane time constant $\tau_{m}$. Grey lines indicate the standard deviation (SD). 
A

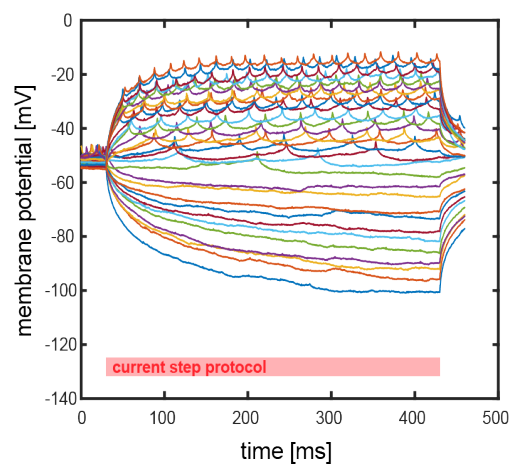

D

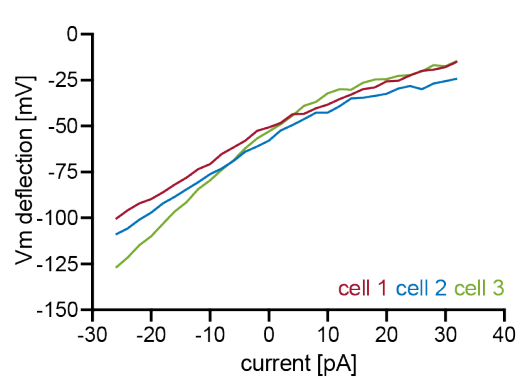

B

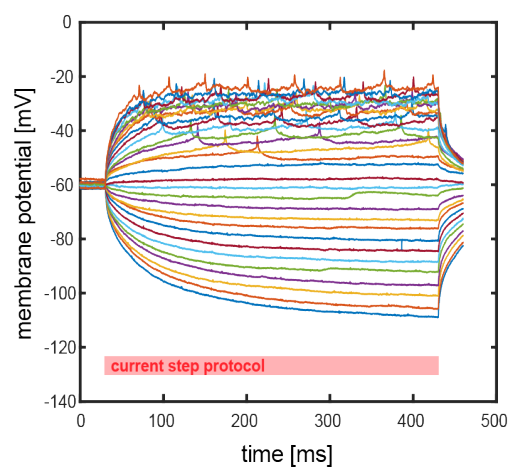

E

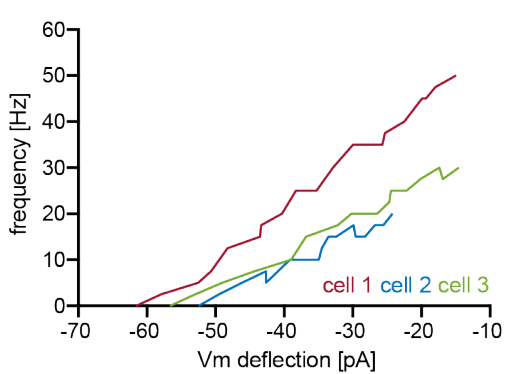

C cell 3

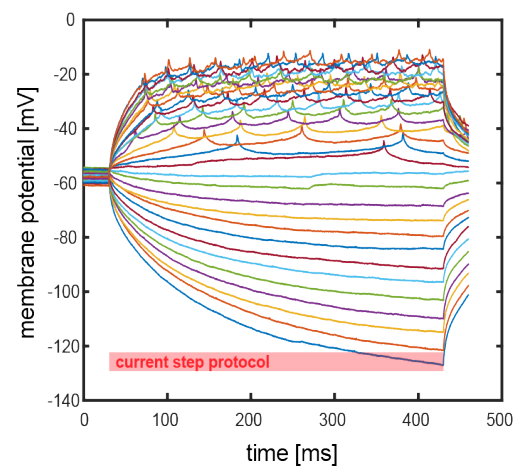

F

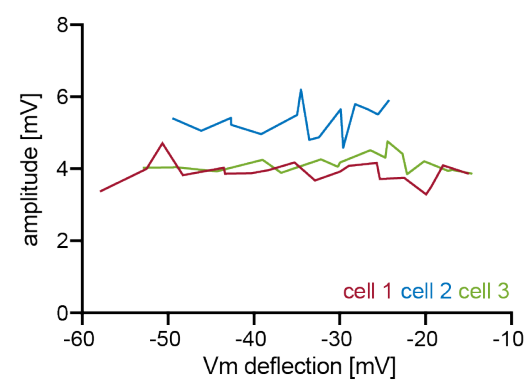

Figure 2: MBON- $\alpha 3$ is a spike frequency adapting neuron. (A) Evoked neuronal activity of MBON$\alpha 3$ through a step-wise increasing injection of $400 \mathrm{~ms}$ current pulses. Pulses start at $-26 \mathrm{pA}$ (bottom) with increasing $2 p A$ steps and end at $+32 p A$ (top). (B) Second example cell. (C) Third example cell. (D) Relative change of membrane potential after current injection. $\Delta \mathrm{Vm}$ is equal to the maximum depolarisation minus resting membrane potential. (E) Correlation between frequency of action potential firing and changes in membrane potential. The frequency describes action potentials per second. (F) Relative change of spike amplitude after current injections. Spike amplitude equals the peak of the highest action potential minus baseline. 
A

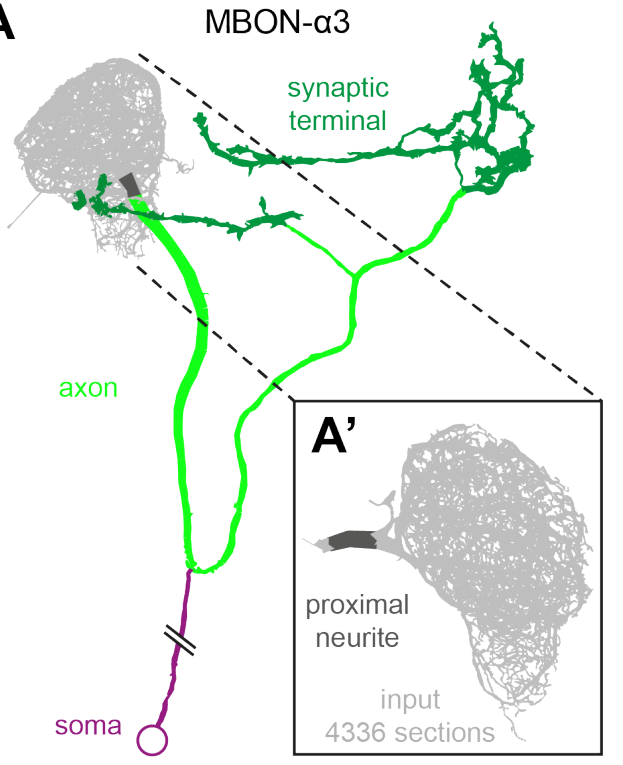

B

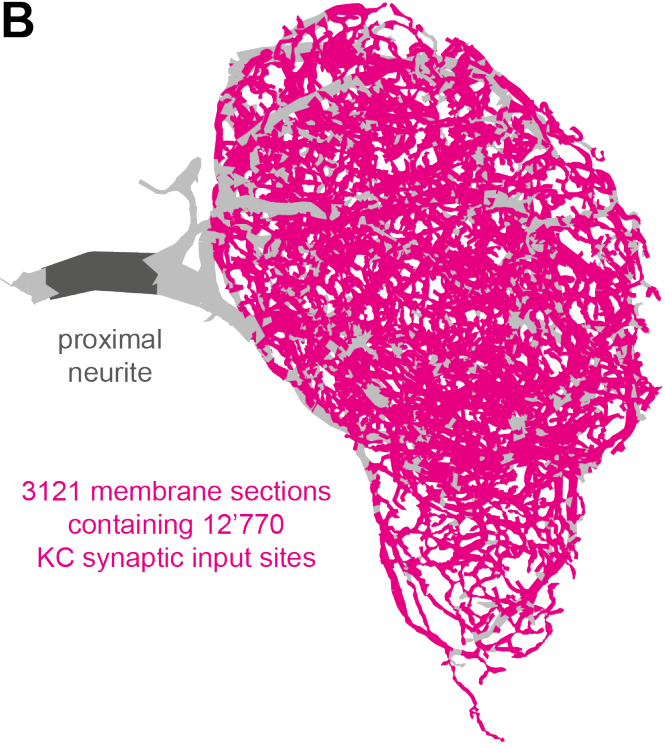

C
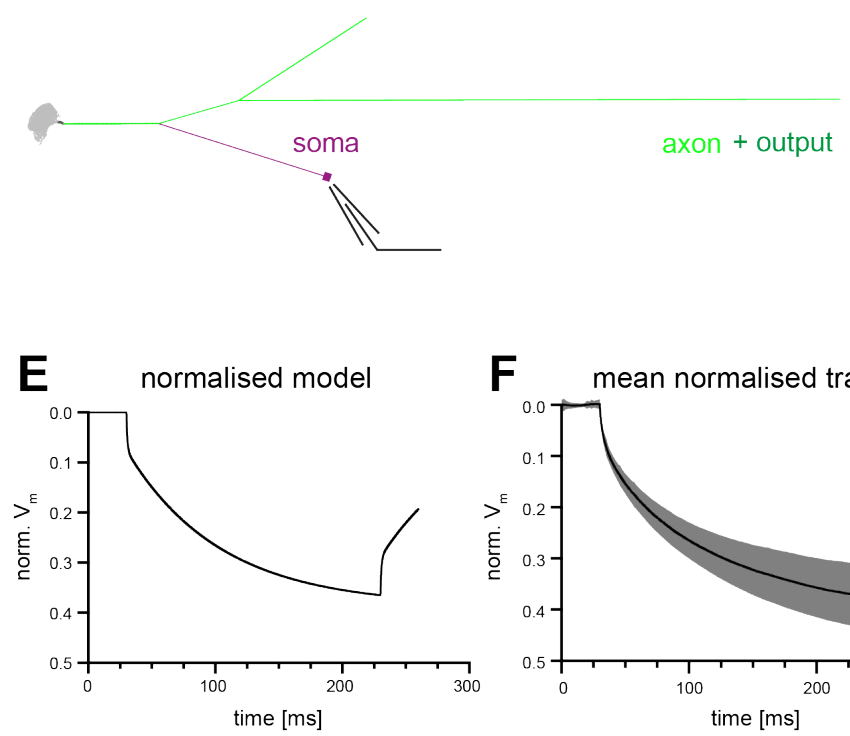

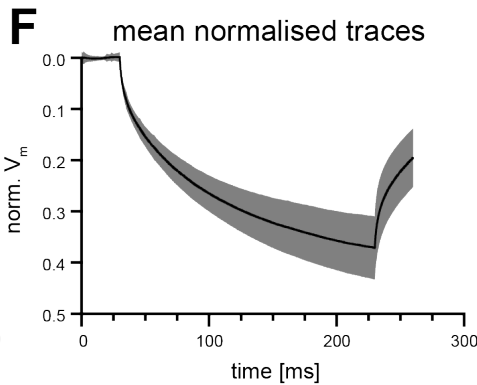

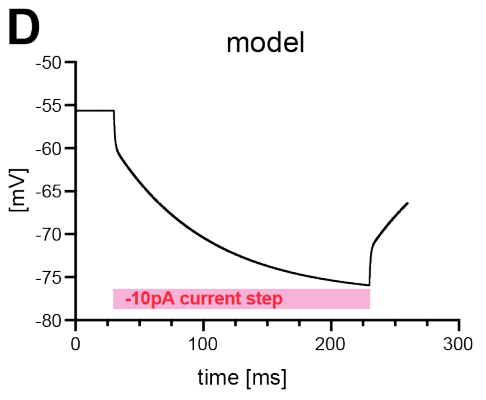

G overlay model and measured

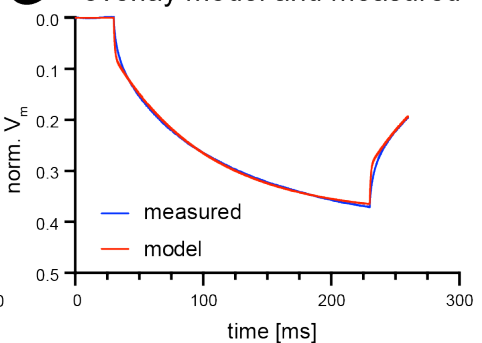

Figure 3: Construction of a computational model of MBON- $\alpha 3$. (A) Electron microscopy based reconstruction of MBON- $\alpha 3$. Data was obtained from NeuPrint [82] and visualised with NEURON [38]. Data set MBON14 (ID 54977) was used for the dendritic architecture (B) and MBON14(a3)R (ID 300972942) was used to model the axon and synaptic terminal (A). The axonal section (light green) connects dendritic input sites (grey) to synaptic terminal (dark green). The connection to the soma (purple) is included for illustration of the overall morphology and is not drawn to scale. (A') Magnified and rotated section of the dendritic input sites of $\mathrm{MBON}-\alpha 3$. The proximal neurite (indicated in dark grey) is the presumed site for action potential generation. Within the dendritic tree a total of 4336 individual membrane sections were defined (light grey area) (B) Magnified section of the dendritic input sites. The 3,121 membrane sections containing 12,770 synaptic input sites from the $948 \mathrm{KCs}$ are highlighted (magenta). (C) In silico model of MBON- $\alpha 3$. We connected 5 neurite segments to the proximal neurite of the dendritic section, corresponding to the segments shown with corresponding colors in (A). These additional segments account for the total length of the axon and synaptic terminal (light green) as well the connection to the soma and the soma itself (purple). Electrophysiological recordings were obtained from the soma; note adjacent symbol of recording electrode. (D) Modelling of an elicited membrane polarization after injection of a $200 \mathrm{~ms}$ long square-pulse current of $-10 \mathrm{pA}$ at the soma, resulting in a depolarization of the membrane potential. (E) Normalised trace of the model. (F) Normalised and averaged traces (black) with (grey) standard deviation of the measured depolarisation, redrawn from Figure $1(\mathrm{G})$. (G) Overlay of normalised induced depolarisation from the model (red) and experiment (blue). The model was fitted to the measured normalised mean traces with a mean squared error between model and measured data of $0.0480917 \mathrm{mV}^{2}$. We performed a two tailed nonparametric Spearman correlation with a confidence interval of $95 \%$ between the two data sets, $r=0.9976$ and $\mathrm{p}=<0.0001$. 
bioRxiv preprint doi: https://doi.org/10.1101/2020.12.06.413500; this version posted March 4, 2021. The copyright holder for this preprint (which was not certified by peer review) is the author/funder. All rights reserved. No reuse allowed without permission.

(Figure $3(\mathrm{E})$ ) and the traces from the in vivo recordings (Figure $3(\mathrm{~F})$ ) and overlayed the resulting traces in Figure $3(\mathrm{G})$. These traces were highly correlated with a Spearman's correlation coefficient of $r=0.9976$. These results indicate that we were able to generate a realistic in silico model of MBON- $\alpha 3$.

\subsection{Effect of KC-MBON synaptic inputs}

As KCs are cholinergic[6] we utilized previously determined parameters of cholinergic synapses to simulate synaptic input to $\mathrm{MBON}-\alpha 3$. We used a reversial potential of $8.9 \mathrm{mV}$ and a synaptic time constant $\tau_{s}=$ $0.44 \mathrm{~ms}$ as target values [78]. Individual synaptic contacts were simulated as alpha functions $\left(g_{\max }(t-\right.$ $\left.\left.t_{i}\right) \times \exp \left(-\left(t-t_{i}-\tau_{s}\right) / \tau_{s}\right)\right)$ where $t_{i}$ is the time of the incoming spike and $g_{\max }=1.56 \times 10^{-11} S$, chosen to obtain response levels in agreement with the target values. The average number of synaptic contacts from a $\mathrm{KC}$ to this $\mathrm{MBON}$ is 13.47 [80], Fig. 5C. To determine the computational constraints of MBON$\alpha 3$ we first stimulated each of the 3,121 dendritic sections that receive $\mathrm{KC}$ synaptic innervation using the described alpha-function conductance change. We then "recorded" (from our simulation) resulting voltage excursions at the dendritic input site (Fig 4A, red), at the proximal neurite (PN, green) and at the cell soma (blue). The comparative analysis of the responses at these three locations revealed two notable features: (1) voltage excursions directly within the dendrite are faster and substantially larger than in the proximal neurite and soma, and (2), the resulting voltages in the latter two compartments are much less variable than in the dendrite and distributed within a very small voltage range (Figure $4 \mathrm{~B}$ and $\mathrm{C}$ ). These results indicate that the morphological architecture and biophysical parameters of MBON- $\alpha 3$ promote a "compactification," or "democratization," of synaptic inputs, with all inputs resulting in similar voltage excursions at the PN and soma, regardless of the strength of the initial dendritic voltage or the position on the dendritic tree.

To evaluate the morphological basis for the input computation we analyzed the relationship between dendritic position, local dendritic volume and resulting amplitude at the dendrite, PN or soma. For these analyses we defined a color code based on the distance between dendritic input and PN or soma, respectively (Figure $4 \mathrm{D}$ ). We observed the largest dendritic amplitudes at distal dendritic sites that have the smallest local section volume (Figure $4 \mathrm{E}$ and $\mathrm{F}$ ). This dendritic architecture with smallest dendritic sections at the most distant sites seems to contribute to the compactness of the dendritic tree that ensures that even the most distant synaptic inputs result in somatic voltage deflections comparable to the most proximal ones. It is important to note again the close range of somatic responses in comparison to the large range of voltages recorded locally at the dendritic input sites (Figure $4 \mathrm{~F}$ ). As a consequence we observed a strong mean attenuation of $75.99 \%$ of the dendritic inputs at the PN and the soma correlating with the distance from the recording site (Figure $4 \mathrm{G}, \mathrm{H}$ ).

We next analyzed the impact of individual KCs on MBON- $\alpha 3$. We simulated activation of all synapses of each individual $\mathrm{KC}$ that innervates $\mathrm{MBON}-\alpha 3$, using the synaptic parameters described above, and we recorded the simulated voltage excursions in the soma, Figure 5 (A). The number of synapses varied between KCs, ranging from 1 to 27 (plus one outlier with 38 synaptic contacts), with a mean of 13.47 synapses per KC Figure 5E, F). In these simulations we observed a wide range of voltage excursions (note the outlier in Figure 5 B,B',C,D,E,F). The neuron is firmly in a small-signal operation mode, as we observed a highly linear relation between the voltage excursion and the number of synapses per KC, Fig 5D. Activating a single KC leads to a voltage excursion at the soma with a mean of $0.37 \mathrm{mV}$, Fig 5C; this value is in good agreement with in vivo data for MBONs [36]. To further analyze this linear relationship between synapse number and somatic voltage excursion, we analyzed all KCs with exactly 13 synaptic inputs to MBON- $\alpha 3$. This analysis revealed a highly stereotypical depolarization at the soma for all these KCs, despite wide variations in the position of the individual synaptic contacts (Figure 5A, E). These results provide further support for the compactness of MBON- $\alpha 3$.

Given that $\approx 5 \%$ of all $984 \mathrm{KCs}$ are typically activated by an odor [14, 39], we next simulated voltage excursions in the soma resulting from the simultaneous activation of sets of 50 randomly selected KCs. On average we activated 673 synapses per trial (Figure 6D, E). In our 1,000 independent activation trials (Figure 6A), we observed highly stereotypical activation patterns, with a mean somatic depolarization of $15.24 \mathrm{mV}$ and only small variations in its time course (Figure $6 \mathrm{~B}, \mathrm{C}$ ). These results are consistent with an activation of $50 \mathrm{KCs}$ being sufficient to induce action potential generation (Fig $2 \mathrm{~A}-\mathrm{C}, \mathrm{E}$ ). The close range of somatic depolarization observed in these experiments supports the finding that odor encoding in KCs is at least partially random (stochastic) [15] as all sets of 50 KCs efficiently induced activation of MBON- $\alpha 3$.

To gain first insights into the sensitivity and potential plasticity mechanisms of the KC-MBON circuitry we repeated the same simulation but now we activated only $75 \%$ of KC-MBON synapses, randomly selected for each of the $50 \mathrm{KCs}$. This experiment addresses in a first, and admittedly simplified approach potential MBON output tuning. In these experiments we observed a significant reduction of somatic 
A

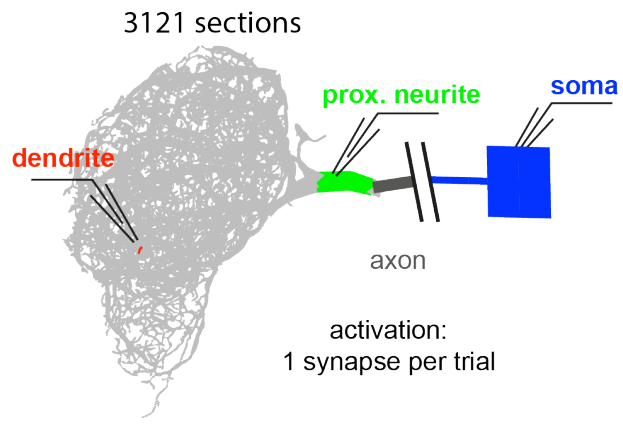

C

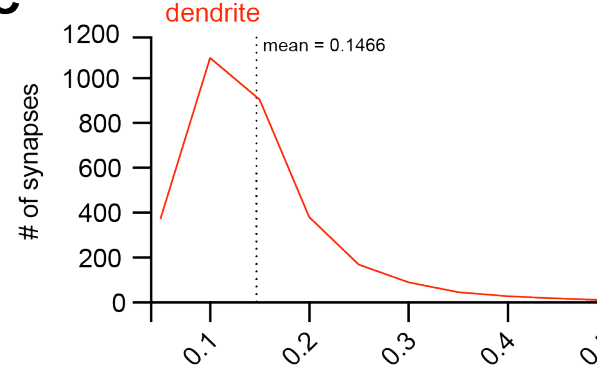

D

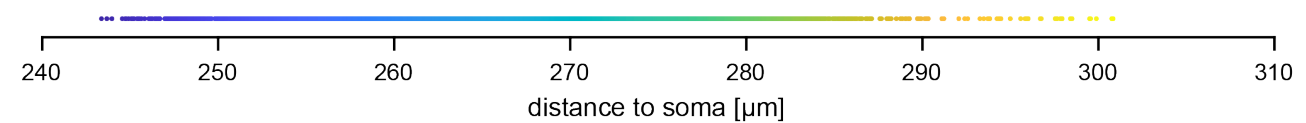

$\mathbf{E}$

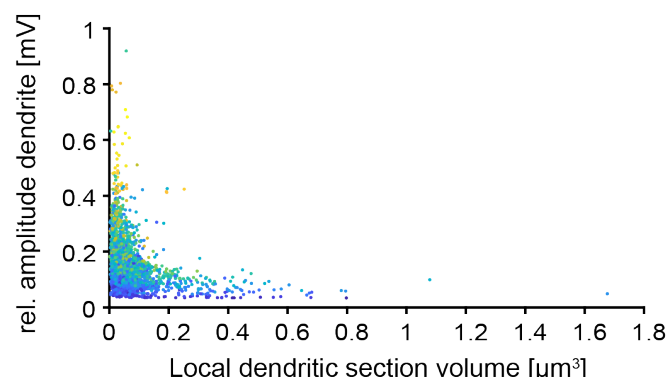

G

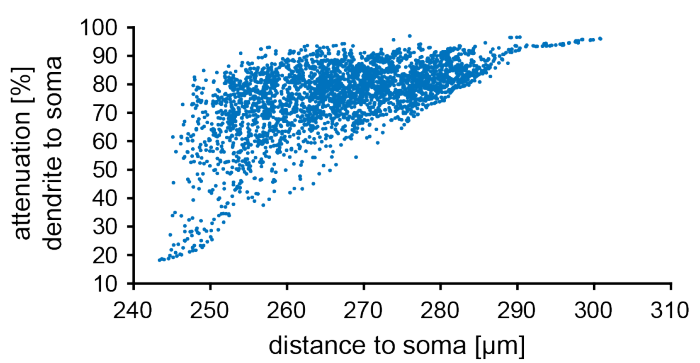

B

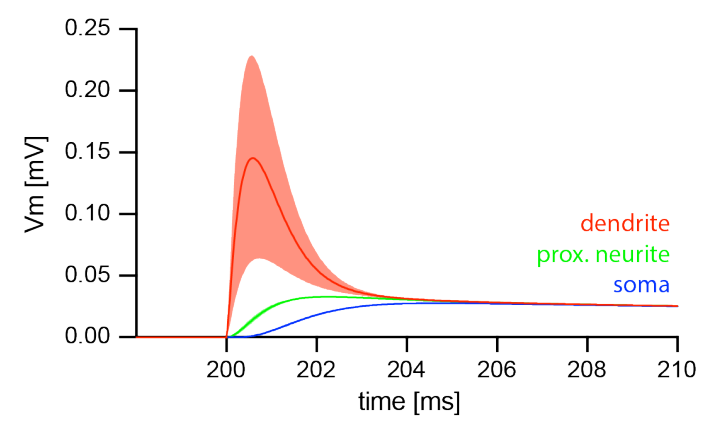

$\mathbf{F}$

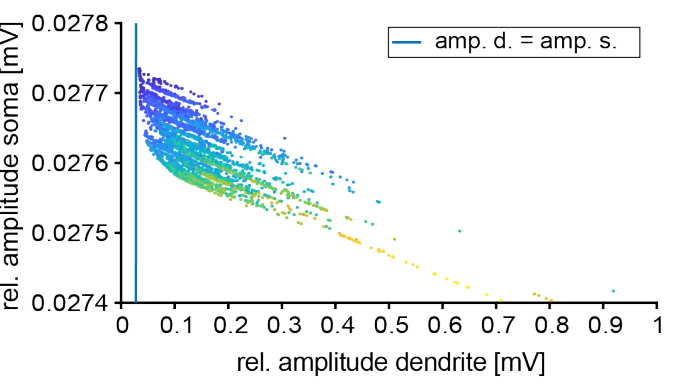

H

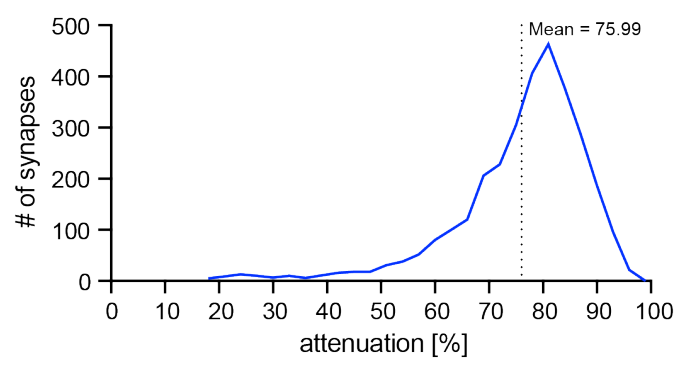

Figure 4: Analysis of voltage attenuation within MBON- $\alpha 3$. (A) Schematic drawing of the simulation experiment. Voltage deflections of synaptic currents were analyzed at the dendritic segment (red), the proximal neurite (green) and the soma (blue). Colors also apply to B and C. (B) Resulting mean voltages (dark lines) and standard deviations (light lines) from 3,121 individual synaptic activations are displayed. (C) Distribution of the measured amplitudes in response to the synaptic activations. Note the difference in scale and compactification of the resulting amplitudes in the proximal neurite and soma (D) Color coded distance distribution for the distance between stimulated dendritic segment and soma. (E) Scatter plot of the elicited relative amplitude in the dendritic section as function of the local dendritic section volume. (F) Scatter plot of the relative soma amplitude as function of the relative amplitude at the dendritic segment. The blue line represents identity. (G) Voltage attenuation at the soma as function of the distance to the dendritic section. (H) Distribution of the percentage of voltage attenuation recorded at the soma. 
A

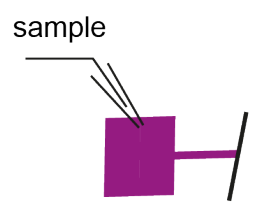

soma
KC 5

(13 synapses)

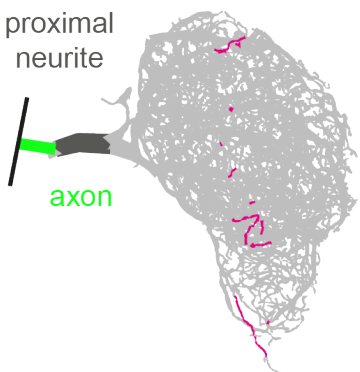

KC 12

(13 synapses)

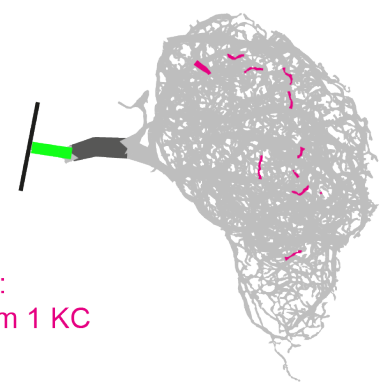

B

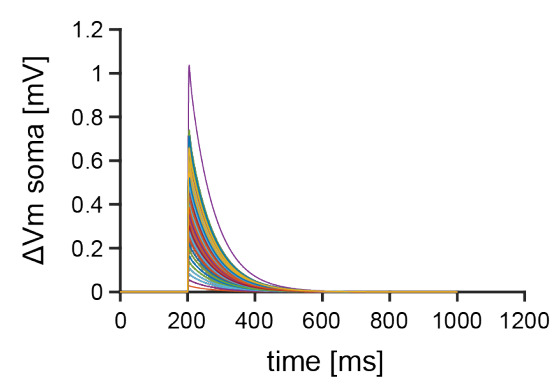

D

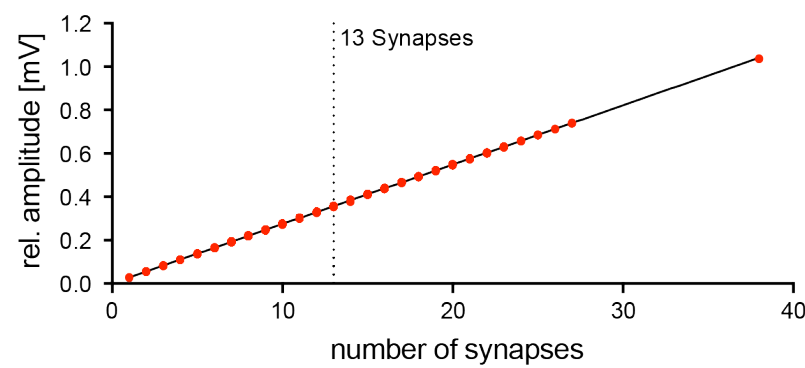

B'

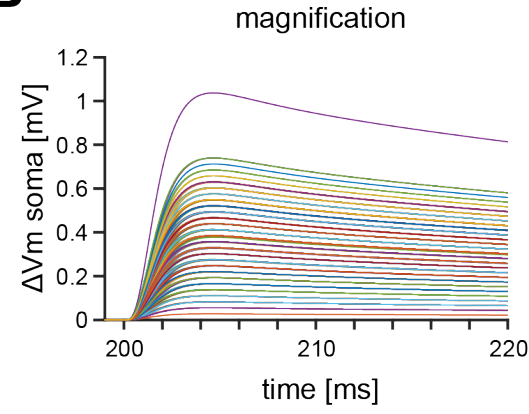

E

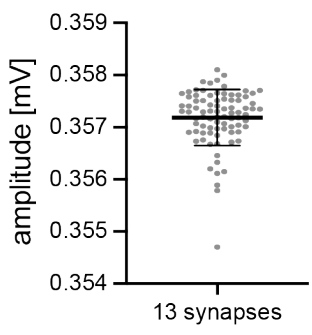

C

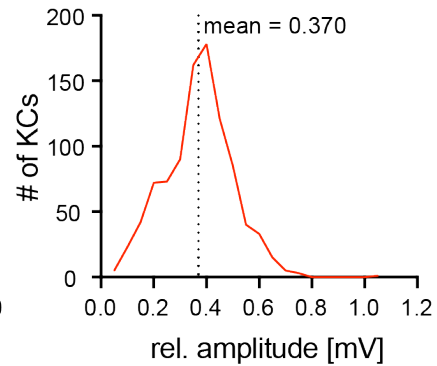

$\mathbf{F}$

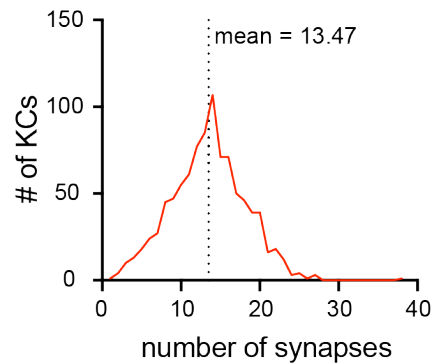

Figure 5: MBON- $\alpha 3$ is electrotonically compact. (A) Schematic of the simulated recordings in the soma (purple) after activation of all individual KCs (magenta - 2 examples with 13 synapses). (B) Membrane potential traces from simulated activation of all $948 \mathrm{KCs}$. (B') Magnification of B. (C) Correlation between the somatic amplitude and the activated KCs. (D) Correlation between the somatic amplitude and the number of activated synapses in KCs. (E) Distribution of the somatic amplitude after the activation of all KCs with 13 synapses. (F) Distribution of the number of synapses per KC. 


\begin{tabular}{|c|c|}
\hline Parameter & Mean \pm SEM \\
\hline \hline$V_{m}[\mathrm{mV}]$ & $-56.7 \pm 2.0$ \\
\hline$\tau_{m}[\mathrm{~ms}]$ & $15.6 \pm 2.3$ \\
\hline$C_{m}[\mathrm{pF}]$ & $16.76 \pm 1.90$ \\
\hline$C_{\text {specific }}\left[\mu \mathrm{F} / \mathrm{cm}^{2}\right]$ & $0.272 \pm 0.031$ \\
\hline$g_{\text {passive }}\left[\mathrm{S} / \mathrm{cm}^{2}\right]$ & $1.78 \times 10^{-5} \pm 0.11 \times 10^{-5}$ \\
\hline
\end{tabular}

Table 1: Summary of passive membrane properties of MBON- $\alpha 3$, calculated from electrophysiological measurements from five neurons. From top to bottom: resting membrane potential $\left(V_{m}\right)$ in $m V$, membrane time constant $\left(\tau_{m}\right)$ in $m s$, capacitance of the membrane $\left(C_{m}\right)$ in $p F$, specific capacitance $\left(C_{\text {specific }}\right)$ in $\mu \mathrm{F} / \mathrm{cm}^{2}$ and passive membrane conductance $\left(g_{\text {passive }}\right)$ in $S / \mathrm{cm}^{2}$.

depolarization that again clustered in a narrow range (Figure 6 B-E). These results are consistent with the clear and almost linear relationship between active number and resulting somatic depolarization (Figure 6 D).

\section{Discussion}

For our understanding of the computational principles underlying learning and memory it is essential to determine the intrinsic contributions of neuronal circuit architecture. Insights into these processes critically depend on realistic simulation of neuronal structure and function. The ability of an animal to adapt its behavior to a large spectrum of sensory information requires specialized neuronal circuit motifs[1]. Associative olfactory memory formation in Drosophila provides a suitable model system to investigate such circuit motifs as a large number of different odors can be associated with either approach or rejection behavior through the formation of long term memory within the MB circuitry. In contrast to most axon guidance processes in Drosophila that are essentially identical in all wild-type individuals, processing of odor information in the MB largely depends on stochastic connectivity of projection neurons to KCs that relay odor information from the olfactory glomeruli to the MBONs $[15,30,48,91]$. Individual flies display idiosyncratic, apparently random connectivity patterns that transmit information of specific odors to the output circuit of the MB. However, the role of MBONs within the circuit is largely fixed between animals and they can be mostly classified as either approach or avoidance neurons $[3,5,46,48,72,81]$. Furthermore, individual KCs are not biased in their MBON connectivity but innervate both kinds of output modules, for both approach and avoidance. Learning and memory, the modulation of odor response behavior through pairing of an individual odor with either a positive or negative valence is incorporated via the local activity of valence-encoding DANs that can either depress or potentiate $\mathrm{KC}>\mathrm{MBON}$ synaptic connections $[4,9,11,11,13,18,21,33,34,41,59,62,66,74,94]$. As $\mathrm{KC}$ odor specificity and connectivity differ significantly between flies with respect to the number and position of $\mathrm{KC}>\mathrm{MBON}$ synapses, this circuit module must be based on architectural features supporting robust formation of multiple parallel memories regardless of specific individual $\mathrm{KC}$ connectivity[41]. By combining precise structural data from the electron microscopy based synaptic connectome [81] of the Drosophila MB with functional data, we were able to generate a realistic in silico model of a mushroom body output neuron that represents a central module for the computation of memory-modulated animal behavior. We based our simulation on vector data recapitulating the neuroanatomical structure of MBON- $\alpha 3$ including appropriate cellular dimensions and positions of all dendritic and axonal compartments, and on the 12,270 synaptic input positions from 948 innervating KCs. Cable theory-based fitting of these data with realistic functional properties of MBON- $\alpha 3$ that we obtained by ex vivo patch clamp recordings allowed us to simulate MBON activation. We restricted our model to passive biophysical parameters including membrane conductance, cytoplasmic resistivity, and capacitance since, to our knowledge, active (nonlinear) amplification mechanisms within the dendritic compartment have not yet been reported in Drosophila. This linear model seems to be an excellent approximation for the dendritic tree, as shown by the close agreement between experimentally observed voltage excursions resulting from controlled current injections and their simulated equivalents.

Going forward, in a more general context, the equations will need to include nonlinear terms in the description of the axonal parts of the neuron. While it is currently unclear whether potential contributions of nonlinear currents play a role in dendrites, it has recently been reported that voltage-gated $\mathrm{Na}^{+}$ channels are localized in axonal initial segment-like regions in central neurons of Drosophila [69]. Thus, excluding from the model active membrane currents generated by interactions between dendrites and axon, including nonlinearities related to action potentials, may result in discrepancies between the model 
bioRxiv preprint doi: https://doi.org/10.1101/2020.12.06.413500; this version posted March 4, 2021. The copyright holder for this preprint (which was not certified by peer review) is the author/funder. All rights reserved. No reuse allowed without permission.

A

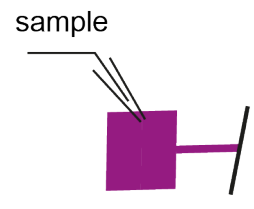

soma trial 1

(50 KCs)

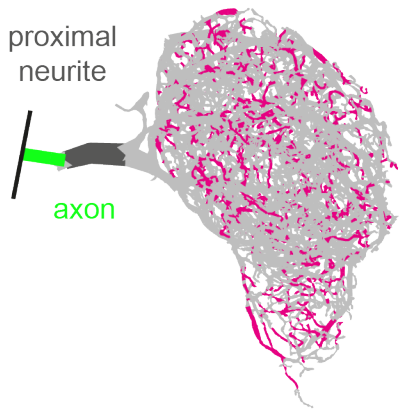

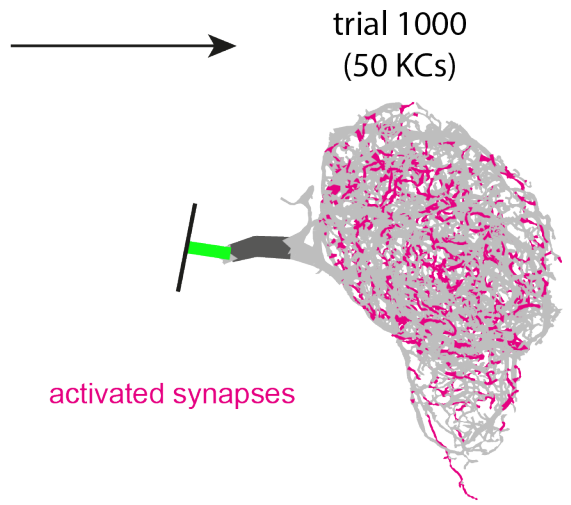

B

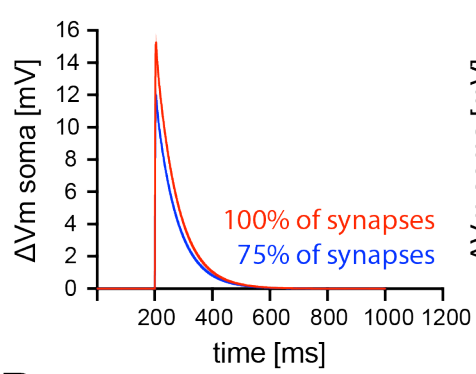

D

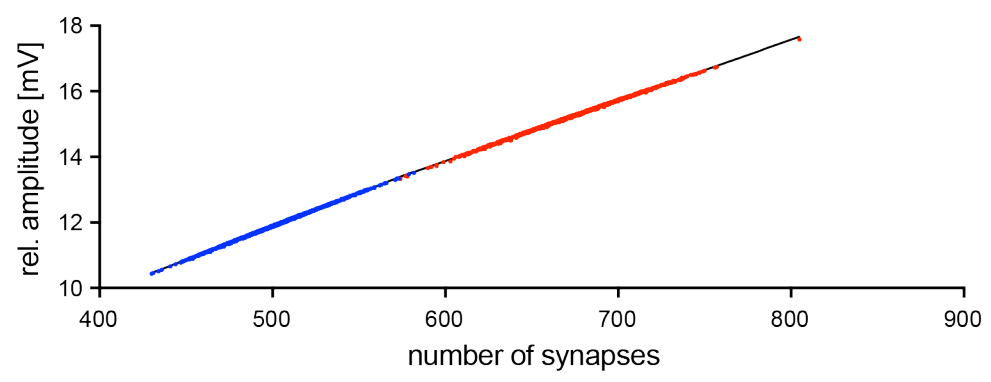

C

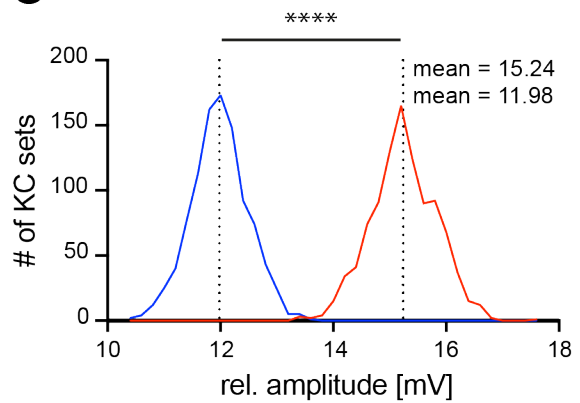

E

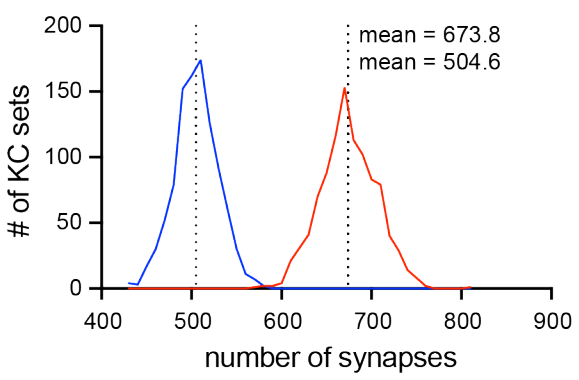

Figure 6: Simulated activations of random combinations of 50 KCs to mimic odor-dependent activation of $\mathrm{MBON}-\alpha 3$. (A) Schematic of the simulated recordings in the soma (purple) and simulated activation patterns of $50 \mathrm{KCs}$ (magenta). Each trial used a different combination of $\mathrm{KCs}$, reflecting acivation patterns of different odors. We activated either all synapses or only $75 \%$ of the synapses. (B, B') Mean somatic voltages and standard deviations after activation of 1000 different sets of 50 KCs with either $100 \%$ (red) or $75 \%$ (blue) of active synapses. Expanded time scale in B'. (C) Distribution of the relative amplitudes evoked by the different KC sets after activation of $100 \%$ (red) or $75 \%$ (blue) of synapses. We observed a significant difference between the two datasets (two-tailed t-test, $p<0.0001$ ) (D) The voltage amplitude at the soma relative to baseline is directly proportional to the number of activated synapses.

(E) Distribution of the number of activated synapses per KC set. Same color code as above. 
and biological reality. Further in vivo recording data will be necessary to quantitatively characterize such local active properties in future implementations of the model.

In contrast to prior simulations of Drosophila neurons [28] our model incorporates the precise locations of all synaptic inputs to the neuron; to the best of our knowledge the only other system in which simulations with comparable level of detail have been published is the nematode C. elegans [57, 58]. Previous studies have suggested that $\operatorname{MBON}-\alpha 3$ is electrotonically compact [16], this is as shown in our simulations at the single synapse level since the location of an individual synaptic input within the dendritic tree has only a very minor effect on the output of this neuron [32]. This was particularly evident when analyzing the results of activating all synapses of all individual $948 \mathrm{KCs}$ innervating MBON- $\alpha 3$. As the number of synaptic inputs ranged from 1 to 28 (with one outlier of 38) we could directly determine the impact of numerical increases in synaptic activation independent of input localization. Amplitudes at the PN or soma scaled almost perfectly linearly with the number of activated synapses. Indeed, when restricting the analysis to a population of KCs with identical numbers of synapses we observed highly stereotypical responses, indicating that differences in synaptic locations do not significantly influence $\mathrm{KC}$ impact. This compactification of the neuron is likely directly related to the architectural structure of the dendritic tree, as inputs to distal dendritic locations induced the largest local depolarizations but corresponding membrane deflections at the proximal neurite and the soma were similar to those elicited by more proximal dendritic locations. Together with the relatively small size of central neurons in Drosophila this indicates that in contrast to large vertebrate neurons local active amplifications or other compensatory mechanisms $[26,27,70,77]$ may not be necessary to support input normalization.

Encoding of odor information and incorporation of memory traces is not performed by individual KCs but by ensembles of KCs. Calcium imaging experiments in vivo demonstrated that individual odors evoke activity in approximately 3-9\% of KCs [14, 39]. Our simulations of 1000 independent trials with random sets of $50 \mathrm{KCs}$, representing approximately $5 \%$ of the KCs in the simulation, demonstrated that such activation patterns robustly elicit MBON activity to a level that matched our in vivo observations of odor induced activity, as well as that found in previously published studies [34, 75, 91]. The low variability of observed depolarizations in these simulations indicates that information coding by such activation patterns is highly robust. As a consequence, hard wiring of odor identity is likely not necessary at the level of KCs as relaying information via any set of $\approx 50 \mathrm{KCs}$ is almost equally efficient.

In our simulations we used the simplest possible activation pattern, with all individual synapses of active KCs providing identical synaptic currents. This approach enabled us to also implement a first model of synaptic plasticity that may underlie memory incorporation in the MB circuit. By activating only a fraction $(75 \%)$ of the synapses of the $50 \mathrm{KCs}$, we simulated selective depression of $\mathrm{KC}>\mathrm{MBON}$ transmission. Optical recordings of in vivo activity of MBONs has revealed selective reductions in MBON activity in response to aversive odor training $[41,61,62,64,74]$ or in response to optogenetic activation of selective DANs [34]. As predicted by our observation of an almost linear correlation between the number of active synapses and the resulting depolarizations, a $25 \%$ reduction of active synapses in the pool of activated KCs significantly decreased the level of MBON activation. Our data would also support potentiation of the $\mathrm{KC}>\mathrm{MBON}$ transmission in response to positive training stimuli as both depression and potentiation of MBON activity have been previously observed at different MBON modules in vivo $[34,41,61,62,64,66,74]$. Our simulation data thus shows that the $\mathrm{KC}>\mathrm{MBON}$ architecture represents a biophysical module that is well-suited to process and store multiple parallel memories based on stochastically encoded odor information provided by KCs.

\section{Materials and methods}

\subsection{Fly Stocks}

Flies were reared on standard fly food at $25^{\circ} \mathrm{C}$ and $65 \%$ humidity. Following stocks were used in this study: 10XUAS-IVS-mCD8::GFP [65] (BDSC 32186), MB082C-Gal4 [2] (BDSC 68286).

\subsection{Immunohistochemistry}

Male flies expressing GFP in MBON- $\alpha 3$ were fixed for $3.5 \mathrm{~h}$ at $4^{\circ} \mathrm{C}$ in $4 \% \mathrm{PFA}$ containing $\mathrm{PBST}(0.2 \%$ Triton-X100). Flies were then washed for at least $3 \times 30 \mathrm{~min}$ at RT in PBST before dissection. Dissected brains were then labelled with primary antibodies (rabbit anti-GFP (A6455, Life technologies, 1:2000) and mouse anti-Brp nc82, Developmental Studies Hybridoma Bank, Iowa, 1:200) for two nights at $4^{\circ} \mathrm{C}$. After incubation, brains were washed at least 6 × 30 min in PBST. Secondary antibodies (Alexa488 (goat $\alpha$ rabbit) and Alexa568 (goat $\alpha$ mouse) coupled antibodies, Life technologies, 1:1000) were applied 
bioRxiv preprint doi: https://doi.org/10.1101/2020.12.06.413500; this version posted March 4, 2021. The copyright holder for this preprint (which was not certified by peer review) is the author/funder. All rights reserved. No reuse allowed without permission.

for two nights at $4^{\circ} \mathrm{C}$. After a repeated washing period, brains were submerged in Vectashield (Vector Laboratories), mounted onto microscope slides and stored at $4^{\circ} \mathrm{C}$.

Images were acquired using a confocal scanning microscope (Zeiss LSM 710) and a 25x (Zeiss PlanNEOFLUAR, NA 0.8 Korr DIC) oil objective. Raw images were projected with Fiji [73] and cropped in Photoshop (Adobe). Uniform adjustments of brightness and contrast were performed.

\subsection{Electrophysiology}

Flies were flipped 1d after hatching to obtain 3-5d old flies. Due to the inconvenient location of the soma we performed ex vivo recordings of the brain. For preparation, flies expressing GFP in MBON$\alpha 3$ were briefly anesthetized on ice before removing the entire brain from the head. The preparation was performed in oxygenated $\left(95 \% \mathrm{O}_{2}\right.$ and $\left.5 \% \mathrm{CO}_{2}\right)$ high glucose $(260 \mathrm{mM})$ extracellular saline. As described previously, the brain was incubated for $30 \mathrm{~s}$ in $0.5 \mathrm{mg} / \mathrm{ml}$ protease (from Streptomyces griseus, CAS\# 90036-06-0, Sigma) containing extracellular saline [92]. The brain was then transferred to standard extracellular saline [92] (in $m \mathrm{M}: 103 \mathrm{NaCl}, 3 \mathrm{KCl}, 5 \mathrm{TES}, 10$ trehalose, 260 glucose, 7 sucrose, 26 $\mathrm{NaHCO}_{3}, 1 \mathrm{NaH}_{2} \mathrm{PO}_{4}, 1.5 \mathrm{CaCl}_{2}, 4 \mathrm{MgCl}_{2}$, pH 7.3, $280-290 \mathrm{mOsmol} / \mathrm{kg}$ adjusted with sucrose). For physiological recordings, the brain was transferred to a glass bottom chamber with continuously perfused $(2 \mathrm{ml} / \mathrm{min})$ oxygenated standard extracellular saline and held in place by a custom made platinum frame. All recordings were performed at room temperature.

We used glass capillaries (GB150 (F-)-10P, Science Products, Hofheim, Germany) and a horizontal puller (Flaming Brown Micropipette Puller P-1000, Sutter instruments, Novato, USA) to obtain pipettes with a resistance of $7-10 M \Omega$. For recordings, patch pipettes were filled with internal solution [52] (in $m M: 140 \mathrm{~K}$-aspartate, $10 \mathrm{HEPES}, 4 \mathrm{Mg}$-ATP, $0.5 \mathrm{Na}$-GTP, 1 EGTA, $1 \mathrm{KCl}$, pH 7.29 adjusted with $\mathrm{KOH}, 265 \mathrm{mOsmol} / \mathrm{kg}$ ). Whole-cell recordings were made using the EPC10 amplifier (HEKA, Germany) and the PatchMaster software (HEKA, Germany). Signals were low-pass filtered at $3 \mathrm{kHz}$, and digitized at $10 \mathrm{kHz}$ via a digital-to-analog converter. The liquid junction potential of $13 \mathrm{mV}$ was corrected online. Patch pipettes were guided under visual control with an upright microscope (BX51WI; Olympus) equipped with a 60x water immersion objective (LUMPlanFl/IR; Olympus) and a CCD camera (Orca 05G, Hamamatsu). GFP signal of target cells was visualized through fluorescence excitation (100W fluorescence lamp, Zeiss, Germany), and a dual-band emission filter set (512/630 HC Dualband Filter GFP/DsRed, AHF Analysentechnik, Germany). We used GFP expression in soma and neurite to identify MBON- $\alpha 3$ neurons. During recordings, the fluorescence excitation was shut off to minimize phototoxic effects. The complete setup was mounted on an air damped table while being shielded by a faraday cage. Series resistance was maintained below $90 M \Omega$ and compensated for up to $35 \%$ through the amplifier's compensation circuitry. In current-clamp mode, cells were held at a baseline of $-60 \mathrm{mV}$ to measure the relevant parameters for the model. Signals were recorded with a sample rate of $20 \mathrm{kHz}$ or $50 \mathrm{kHz}$ and low-pass filtered at $5 \mathrm{kHz}$.

To determine passive electrical membrane properties and qualitative patch characteristics a series of standard protocols were performed:

VCstep without compensation. In voltage-clamp mode, a voltage-step $(100 \mathrm{~ms}, 5 \mathrm{mV})$ is applied without compensating the series resistance. The corresponding current trace was then used to determine $R_{\text {series }}, R_{\text {input }}$ and $R_{m}$ with Igor Pro (WaveMetrics).

CCdehyper. In current-clamp mode, a step-protocol was performed and recorded at $50 \mathrm{kHz}$. Each sweep starts with $0 p A$ for $10 \mathrm{~ms}$, which is followed by a current injection $(-26 p A$ to $32 p A$, with $2 p A$ increments) and a step duration of $400 \mathrm{~ms}$. The resulting changes in membrane potential and induced action potentials were then analyzed and plotted with Matlab (MathWorks). The maximum depolarisation was calculated as a mean of the last 70 sample points of the stimulation in each trace. The resting membrane potential was calculated as the mean of the first 500 sample points before the stimulation in each trace. The absolute action potential was measured as maximum of each trace. The relative action potential amplitude resulted from subtracting the baseline from the absolute value. The baseline resulted from the mean of a 400 sample points window, starting 700 sample points upstream of the maxima in each trace.

CCcontinuous. In current-clamp mode, no current was injected through out a $60 \mathrm{~s}$ recording at $50 \mathrm{kHz}$. The resting membrane potential was determined as the mean of the baseline in the recording with Igor Pro and spontaneous activity was plotted with Matlab.

$C$ Cinject. In current-clamp mode, a short current pulse $(-50 p A, 100 \mathrm{~ms})$ was injected and recorded at $20 \mathrm{kHz}$. We performed 35 iterations per cell. The resulting change in membrane potential was used to determine $\tau_{m}$ with a single exponential fit in Igor Pro. Averaging, normalisation $\left(V_{m}-V_{m 0} / V_{m 0}\right)$ and plotting was performed with Matlab. This data was used to perform the model fitting. The baseline $\left(V_{m 0}\right)$ resulted from the average of the sample points before stimulation. 
Cells displaying a resting membrane potential higher than $-45 \mathrm{mV}$ and/or a $R_{m}$ smaller than $600 M \Omega$ were excluded from the analysis.

\subsection{Computational Model}

Morphology data for the Drosophila MBON- $\alpha 3$-A was originally characterized using scanning electron microscopy [82] and for the present study obtained from the "mushroombody" dataset hosted on the database neuPrint (https://neuprint-examples.janelia.org/; cell ID 54977). These data describe the structure of its dendritic arborization throughout the MBON $\alpha 3$ compartment (Fig. 3A'), with coordinates specified at $8 \mathrm{~nm}$ pixel resolution and converted to $\mu \mathrm{m}$ for model implementation. The reconstruction is limited to the portion of the neuron in the mushroom body, not including its soma and axon. The geometry of the axon was determined by investigating a separate MBON, MBON14(a3)R (Fig. 3A), obtained from neuPrint's "hemibrain:v1.1" dataset (https://neuprint.janelia.org/; cell ID 300972942). The axon and synaptic terminal of this neuron were approximated as five separate sections, distinguished by the major branch points identified in the electron microscopic reconstruction. Each section was characterized by its total length and average diameter and divided into segments to maintain the average section length of that region in the original reconstruction (Fig. S3). They were then appended to the MBON- $\alpha 3-\mathrm{A}$ proximal neurite as individual linear segments. The dimensions of the soma were characterized by our light microscopic images of MBON- $\alpha 3-\mathrm{A}$ (Fig. 1A). Synaptic locations of the KC innervation of this MBON- $\alpha 3-\mathrm{A}$ were also obtained from neuPrint's "mushroombody" dataset. MBON- $\alpha 3-\mathrm{A}$ is innervated by 948 distinct $\mathrm{KCs}$, forming a total of 12,770 synapses with an average of 13.47 synapses per KC [82].

Electrophysiological properties of the neuron were determined by fitting the model response to recorded data, an approach consistent with prior work modeling Drosophila central neurons [28]. Somatic voltage excursions in response to $200 \mathrm{~ms}$ current injections of $-10 \mathrm{pA}$ were recorded in vivo (Fig. $1 \mathrm{G}$ ). This current injection was replicated in silico and the membrane potential change at the soma was fit to our recorded data by varying the cell's cytoplasm resistivity, specific membrane capacitance, and passive membrane conductance. The fitting was performed using NEURON's principle axis optimization algorithm, where parameters were tuned until the computed voltage excursion at the soma matched our electrophysiological recordings. The model optimization searched parameter space to minimize an error function defined as the square differences between the averaged experimental responses to current pulses (Fig. $1 \mathrm{G}$ ) and the computed model responses (Fig. 3D). The fitting was then verified by normalizing both the model and experimental voltage excursions and performing two-tailed nonparametric Spearman correlation. The model was first allowed to equilibrate for $30 \mathrm{~ms}$ simulated time, after which the $200 \mathrm{~ms}$ current pulse was initiated. The region of data used for the the fitting ranged from $32.025 \mathrm{~ms}$ to $228.03 \mathrm{~ms}$, excluding approximately the first and last $2 \mathrm{~ms}$ of current injection. The domain of the parameter space was restricted to physiologically plausible values: $0.5 \mu \mathrm{F} / \mathrm{cm}^{2}$ to $1.5 \mu \mathrm{F} / \mathrm{cm}^{2}$ for specific membrane capacitance, $1 \mathrm{E}-7 \mathrm{~S} / \mathrm{cm}^{2}$ to $1 \mathrm{E}-4 \mathrm{~S} / \mathrm{cm}^{2}$ for passive membrane conductance, and $30 \Omega \mathrm{cm}$ to $400 \Omega \mathrm{cm}$ for cytoplasm resistivity. The final values for each parameter (Table S3) are well within these margins. Resting membrane potential and the leak current reversal potential were set at $-55.64 \mathrm{mV}$ based on the initial conditions of the recorded data to which the model was fit.

Synapses were modeled as localized conductance changes described by alpha functions. KC innervation of MBON- $\alpha 3-\mathrm{A}$ was determined to be cholinergic [82]. Currents recorded in Drosophila KCs were demonstrated to have a reversal potential of $8.9 \mathrm{mV}$ and a rise time to maximal conductance of $0.44 \mathrm{~ms}$ [78]. The maximal conductance for the synapses was set at $1.5627 * 10^{-5} \mu S$, the value determined to achieve the target MBON depolarization from monosynaptic KC innervation [36].

All simulations were performed using the NEURON 7.7.2 simulation environment [38] running through a Python 3.8.5 interface in Ubuntu 20.04.1. The morphology data divided the MBON- $\alpha 3-\mathrm{A}$ dendritic tree into 4336 small sections averaging $1.03 \mu \mathrm{m}$ in length and $0.29 \mu \mathrm{m}$ in diameter. With the added sections describing the axon, synaptic terminals, and soma, the model contained 4342 sections averaging $1.24 \mu \mathrm{m}$ in length and $0.29 \mu \mathrm{m}$ in diameter. Each dendritic section was automatically assigned an index number by NEURON. Each of these sections was modeled as a piece of cylindrical cable, with connectivity specified by the neuron's morphology. Passive leak channels were inserted along the membrane of the dendrite sections as well as throughout the axon and soma. The coordinate location of each KC synapse was mapped to the nearest section of the MBON dendrite, and the conductance-based synapse model was implemented in the center of that section. Simulations were performed in which each synapse-containing section was individually activated, followed by additional simulations activating groups of synapses corresponding to single or multiple active KCs. Membrane potential data was analyzed and plotted with MATLAB R2020b (MathWorks) and Prism (GraphPad). 
bioRxiv preprint doi: https://doi.org/10.1101/2020.12.06.413500; this version posted March 4, 2021. The copyright holder for this preprint (which was not certified by peer review) is the author/funder. All rights reserved. No reuse allowed without permission.

\section{Acknowledgments}

This work was supported by NSF 1835202, NIH R01DA040990 and NIH R01EY027544. We would like to thank Glen Turner and Toshihide Hige for insightful comments to the patch clamp analysis. 
bioRxiv preprint doi: https://doi.org/10.1101/2020.12.06.413500; this version posted March 4, 2021. The copyright holder for this preprint (which was not certified by peer review) is the author/funder. All rights reserved. No reuse allowed without permission.

\section{References}

[1] Paul Ardin, Fei Peng, Michael Mangan, Konstantinos Lagogiannis, and Barbara Webb. Using an Insect Mushroom Body Circuit to Encode Route Memory in Complex Natural Environments. PLOS Computational Biology, 12(2):e1004683, February 2016.

[2] Yoshinori Aso, Daisuke Hattori, Yang Yu, Rebecca M Johnston, Nirmala A Iyer, Teri-TB Ngo, Heather Dionne, L F Abbott, Richard Axel, Hiromu Tanimoto, and Gerald M Rubin. The neuronal architecture of the mushroom body provides a logic for associative learning. eLife, 3:e10806, December 2014.

[3] Yoshinori Aso, Daisuke Hattori, Yang Yu, Rebecca M Johnston, Nirmala A Iyer, Teri-TB Ngo, Heather Dionne, Lf Abbott, Richard Axel, Hiromu Tanimoto, and Gerald M Rubin. The neuronal architecture of the mushroom body provides a logic for associative learning. eLife, 3:e04577, December 2014 .

[4] Yoshinori Aso and Gerald M Rubin. Dopaminergic neurons write and update memories with celltype-specific rules. eLife, 5:e16135, July 2016.

[5] Yoshinori Aso, Divya Sitaraman, Toshiharu Ichinose, Karla R Kaun, Katrin Vogt, Ghislain Belliart-Guérin, Pierre-Yves Plaçais, Alice A Robie, Nobuhiro Yamagata, Christopher Schnaitmann, William J Rowell, Rebecca M Johnston, Teri-T B Ngo, Nan Chen, Wyatt Korff, Michael N Nitabach, Ulrike Heberlein, Thomas Preat, Kristin M Branson, Hiromu Tanimoto, and Gerald M Rubin. Mushroom body output neurons encode valence and guide memory-based action selection in Drosophila. eLife, 3:e04580, December 2014.

[6] Oliver Barnstedt, David Owald, Johannes Felsenberg, Ruth Brain, John-Paul Moszynski, Clifford B. Talbot, Paola N. Perrat, and Scott Waddell. Memory-Relevant Mushroom Body Output Synapses Are Cholinergic. Neuron, 89(6):1237-1247, March 2016.

[7] C.G. Becker, A. Artola, R. Gerardy-Schahn, T. Becker, H. Welzl, and M. Schachner. The polysialic acid modification of the neural cell adhesion molecule is involved in spatial learning and hippocampal long-term potentiation. Journal of Neuroscience Research, 45:143-152, 1996.

[8] Sandra D. Berger and Sharon M. Crook. Modeling the influence of ion channels on neuron dynamics in drosophila. Frontiers in Computational Neuroscience, 9:139, 2015.

[9] Jacob A. Berry, Anna Phan, and Ronald L. Davis. Dopamine Neurons Mediate Learning and Forgetting through Bidirectional Modulation of a Memory Trace. Cell Reports, 25(3):651-662.e5, October 2018.

[10] Alexander Borst and Juergen Haag. The intrinsic electrophysiological characteristics of fly lobula plate tangential cells: I. Passive membrane properties. Journal of Computational Neuroscience, 3(4):313-336, December 1996.

[11] Emna Bouzaiane, Séverine Trannoy, Lisa Scheunemann, Pierre-Yves Plaçais, and Thomas Preat. Two Independent Mushroom Body Output Circuits Retrieve the Six Discrete Components of Drosophila Aversive Memory. Cell Reports, 11(8):1280-1292, May 2015.

[12] Christopher J Burke, Wolf Huetteroth, David Owald, Emmanuel Perisse, Michael J Krashes, Gaurav Das, Daryl Gohl, Marion Silies, Sarah Certel, and Scott Waddell. Layered reward signalling through octopamine and dopamine in Drosophila. Nature, 492(7429):433-437, October 2012.

[13] Christopher J. Burke, Wolf Huetteroth, David Owald, Emmanuel Perisse, Michael J. Krashes, Gaurav Das, Daryl Gohl, Marion Silies, Sarah Certel, and Scott Waddell. Layered reward signalling through octopamine and dopamine in Drosophila. Nature, 492(7429):433-437, December 2012.

[14] R. A. A. Campbell, K. S. Honegger, H. Qin, W. Li, E. Demir, and G. C. Turner. Imaging a Population Code for Odor Identity in the Drosophila Mushroom Body. Journal of Neuroscience, 33(25):10568-10581, June 2013.

[15] Sophie J. C. Caron, Vanessa Ruta, L. F. Abbott, and Richard Axel. Random convergence of olfactory inputs in the Drosophila mushroom body. Nature, 497(7447):113-117, May 2013.

[16] Stijn Cassenaer and Gilles Laurent. Hebbian stdp in mushroom bodies facilitates the synchronous flow of olfactory information in locusts. Nature, 448(7154):709, 2007. 
[17] Stijn Cassenaer and Gilles Laurent. Hebbian STDP in mushroom bodies facilitates the synchronous flow of olfactory information in locusts. Nature, 448(7154):709-713, August 2007.

[18] Ann-Shyn Chiang, Chih-Yung Lin, Chao-Chun Chuang, Hsiu-Ming Chang, Chang-Huain Hsieh, Chang-Wei Yeh, Chi-Tin Shih, Jian-Jheng Wu, Guo-Tzau Wang, Yung-Chang Chen, Cheng-Chi Wu, Guan-Yu Chen, Yu-Tai Ching, Ping-Chang Lee, Chih-Yang Lin, Hui-Hao Lin, Chia-Chou Wu, HaoWei Hsu, Yun-Ann Huang, Jing-Yi Chen, Hsin-Jung Chiang, Chun-Fang Lu, Ru-Fen Ni, Chao-Yuan Yeh, and Jenn-Kang Hwang. Three-Dimensional Reconstruction of Brain-wide Wiring Networks in Drosophila at Single-Cell Resolution. Current Biology, 21(1):1-11, January 2011.

[19] Paola Cognigni, Johannes Felsenberg, and Scott Waddell. Do the right thing: neural network mechanisms of memory formation, expression and update in Drosophila. Current opinion in neurobiology, 49:51-58, April 2018.

[20] Raphael Cohn, Ianessa Morantte, and Vanessa Ruta. Coordinated and Compartmentalized Neuromodulation Shapes Sensory Processing in Drosophila. Cell, 163(7):1742-1755, December 2015.

[21] Raphael Cohn, Ianessa Morantte, and Vanessa Ruta. Coordinated and Compartmentalized Neuromodulation Shapes Sensory Processing in Drosophila. Cell, 163(7):1742-1755, December 2015.

[22] J. de Belle and M Heisenberg. Associative odor learning in Drosophila abolished by chemical ablation of mushroom bodies. Science, 263(5147):692-695, February 1994.

[23] Georg Dietzl, Doris Chen, Frank Schnorrer, Kuan-Chung Su, Yulia Barinova, Michaela Fellner, Beate Gasser, Kaolin Kinsey, Silvia Oppel, Susanne Scheiblauer, Africa Couto, Vincent Marra, Krystyna Keleman, and Barry J Dickson. A genome-wide transgenic RNAi library for conditional gene inactivation in Drosophila. Nature, 448(7150):151-156, July 2007.

[24] Johannes Felsenberg, Pedro F. Jacob, Thomas Walker, Oliver Barnstedt, Amelia J. EdmondsonStait, Markus W. Pleijzier, Nils Otto, Philipp Schlegel, Nadiya Sharifi, Emmanuel Perisse, Carlas S. Smith, J. Scott Lauritzen, Marta Costa, Gregory S.X.E. Jefferis, Davi D. Bock, and Scott Waddell. Integration of Parallel Opposing Memories Underlies Memory Extinction. Cell, 175(3):709-722.e15, October 2018

[25] Florence Friggi-Grelin, H 1 ne Coulom, Margaret Meller, Delphine Gomez, Jay Hirsh, and Serge Birman. Targeted gene expression inDrosophila dopaminergic cells using regulatory sequences from tyrosine hydroxylase. Journal of Neurobiology, 54(4):618-627, January 2003.

[26] Robert C Froemke, Mu-ming Poo, and Yang Dan. Spike-timing-dependent synaptic plasticity depends on dendritic location. Nature, 434(7030):221-225, 2005.

[27] Albert Gidon and Idan Segev. Spike-timing-dependent synaptic plasticity and synaptic democracy in dendrites. Journal of neurophysiology, 101(6):3226-3234, 2009.

[28] N. W. Gouwens and R. I. Wilson. Signal Propagation in Drosophila Central Neurons. Journal of Neuroscience, 29(19):6239-6249, May 2009.

[29] Bernd Grünewald. Differential expression of voltage-sensitive K+ and Ca2+ currents in neurons of the honeybee olfactory pathway. The Journal of Experimental Biology, 206(Pt 1):117-129, January 2003.

[30] Eyal Gruntman and Glenn C Turner. Integration of the olfactory code across dendritic claws of single mushroom body neurons. Nature Neuroscience, 16(12):1821-1829, December 2013.

[31] Cicely X Gu, Peter F Juranka, and Catherine E Morris. Stretch-activation and stretch-inactivation of Shaker-IR, a voltage-gated K+ channel. Biophysical Journal, 80(6):2678-2693, 2001.

[32] Omar Hafez, Benjamin Escribano, Jan Pielage, and Ernst Niebur. Integration of Odor-Induced Activity of Kenyon Cells in an Electrotonically Compact Drosophila Mushroom Body Output Neuron (MBON). Preprint, Neuroscience, November 2019.

[33] Annie Handler, Thomas G.W. Graham, Raphael Cohn, Ianessa Morantte, Andrew F. Siliciano, Jianzhi Zeng, Yulong Li, and Vanessa Ruta. Distinct Dopamine Receptor Pathways Underlie the Temporal Sensitivity of Associative Learning. Cell, 178(1):60-75.e19, June 2019. 
[34] Toshihide Hige, Yoshinori Aso, Mehrab N. Modi, Gerald M. Rubin, and Glenn C. Turner. Heterosynaptic Plasticity Underlies Aversive Olfactory Learning in Drosophila. Neuron, 88(5):985-998, December 2015.

[35] Toshihide Hige, Yoshinori Aso, Mehrab N Modi, Gerald M Rubin, and Glenn C Turner. Heterosynaptic Plasticity Underlies Aversive Olfactory Learning in Drosophila. Neuron, 88(5):985-998, December 2015.

[36] Toshihide Hige, Yoshinori Aso, Gerald M. Rubin, and Glenn C. Turner. Plasticity-driven individualization of olfactory coding in mushroom body output neurons. Nature, 526(7572):258-262, October 2015.

[37] Toshihide Hige, Yoshinori Aso, Gerald M Rubin, and Glenn C Turner. Plasticity-driven individualization of olfactory coding in mushroom body output neurons. Nature, 526(7572):258-262, September 2015 .

[38] M. L. Hines and N. T. Carnevale. The Neuron Simulation Environment. Neural Computation, 9(6), Aug. 1997.

[39] K. S. Honegger, R. A. A. Campbell, and G. C. Turner. Cellular-Resolution Population Imaging Reveals Robust Sparse Coding in the Drosophila Mushroom Body. Journal of Neuroscience, 31(33):11772-11785, August 2011.

[40] Wolf Huetteroth, Emmanuel Perisse, Suewei Lin, Martín Klappenbach, Christopher Burke, and Scott Waddell. Sweet Taste and Nutrient Value Subdivide Rewarding Dopaminergic Neurons in Drosophila. Current Biology, 25(6):751-758, March 2015.

[41] Pedro F. Jacob and Scott Waddell. Spaced Training Forms Complementary Long-Term Memories of Opposite Valence in Drosophila. Neuron, page S0896627320302208, April 2020.

[42] Arnim Jenett, Gerald M Rubin, Teri-T B Ngo, David Shepherd, Christine Murphy, Heather Dionne, Barret D Pfeiffer, Amanda Cavallaro, Donald Hall, Jennifer Jeter, Nirmala Iyer, Dona Fetter, Joanna H Hausenfluck, Hanchuan Peng, Eric T Trautman, Robert R Svirskas, Eugene W Myers, Zbigniew R Iwinski, Yoshinori Aso, Gina M DePasquale, Adrianne Enos, Phuson Hulamm, Shing Chun Benny Lam, Hsing-Hsi Li, Todd R Laverty, Fuhui Long, Lei Qu, Sean D Murphy, Konrad Rokicki, Todd Safford, Kshiti Shaw, Julie H Simpson, Allison Sowell, Susana Tae, Yang Yu, and Christopher T Zugates. A GAL4-Driver Line Resource for Drosophila Neurobiology. Cell reports, 2(4):991-1001, October 2012.

[43] Colleen Kirkhart and Kristin Scott. Gustatory Learning and Processing in the Drosophila Mushroom Bodies. The Journal of Neuroscience, 35(15):5950-5958, April 2015.

[44] Nathan C Klapoetke, Yasunobu Murata, Sung Soo Kim, Stefan R Pulver, Amanda Birdsey-Benson, Yong Ku Cho, Tania K Morimoto, Amy S Chuong, Eric J Carpenter, Zhijian Tian, Jun Wang, Yinlong Xie, Zhixiang Yan, Yong Zhang, Brian Y Chow, Barbara Surek, Michael Melkonian, Vivek Jayaraman, Martha Constantine-Paton, Gane Ka-Shu Wong, and Edward S Boyden. Independent optical excitation of distinct neural populations. Nature Methods, 11(3):338-346, February 2014.

[45] Sen-Lin Lai and Tzumin Lee. Genetic mosaic with dual binary transcriptional systems in Drosophila. Nature Neuroscience, 9(5):703-709, April 2006.

[46] Feng Li, Jack W Lindsey, Elizabeth C Marin, Nils Otto, Marisa Dreher, Georgia Dempsey, Ildiko Stark, Alexander S Bates, Markus William Pleijzier, Philipp Schlegel, Aljoscha Nern, Shin-ya Takemura, Nils Eckstein, Tansy Yang, Audrey Francis, Amalia Braun, Ruchi Parekh, Marta Costa, Louis K Scheffer, Yoshi Aso, Gregory SXE Jefferis, Larry F Abbott, Ashok Litwin-Kumar, Scott Waddell, and Gerald M Rubin. The connectome of the adult Drosophila mushroom body provides insights into function. eLife, 9:e62576, December 2020.

[47] Hui-Hao Lin, Jason Sih-Yu Lai, An-Lun Chin, Yung-Chang Chen, and Ann-Shyn Chiang. A Map of Olfactory Representation in the Drosophila Mushroom Body. Cell, 128(6):1205-1217, March 2007.

[48] Ashok Litwin-Kumar, Kameron Decker Harris, Richard Axel, Haim Sompolinsky, and L.F. Abbott. Optimal Degrees of Synaptic Connectivity. Neuron, 93(5):1153-1164.e7, March 2017. 
[49] Margaret S. Livingstone, Patricia P. Sziber, and William G. Quinn. Loss of calcium/calmodulin responsiveness in adenylate cyclase of rutabaga, a Drosophila learning mutant. Cell, 37(1):205-215, May 1984.

[50] Lindsey J Macpherson, Emanuela E Zaharieva, Patrick J Kearney, Michael H Alpert, Tzu-Yang Lin, Zeynep Turan, Chi-Hon Lee, and Marco Gallio. Dynamic labelling of neural connections in multiple colours by trans-synaptic fluorescence complementation. Nature communications, 6(1):501, December 2015.

[51] P. Masek and K. Scott. Limited taste discrimination in Drosophila. Proceedings of the National Academy of Sciences, 107(33):14833-14838, August 2010.

[52] A. S. Mauss, M. Meier, E. Serbe, and A. Borst. Optogenetic and Pharmacologic Dissection of Feedforward Inhibition in Drosophila Motion Vision. Journal of Neuroscience, 34(6):2254-2263, February 2014.

[53] Sean M.J McBride, Giovanna Giuliani, Catherine Choi, Paul Krause, Dana Correale, Karli Watson, Glenn Baker, and Kathleen K Siwicki. Mushroom Body Ablation Impairs Short-Term Memory and Long-Term Memory of Courtship Conditioning in Drosophila melanogaster. Neuron, 24(4):967-977, December 1999.

[54] Tomoyuki Miyashita, Emi Kikuchi, Junjiro Horiuchi, and Minoru Saitoe. Long-Term Memory Engram Cells Are Established by c-Fos/CREB Transcriptional Cycling. Cell Reports, 25(10):27162728.e3, December 2018.

[55] E. Niebur. Electrical properties of cell membranes. Scholarpedia, 3(6):7166, 2008.

[56] E. Niebur. Neuronal Cable Theory. Scholarpedia, 3(5):2674, 2008.

[57] E. Niebur and P. Erdős. Modeling Locomotion and its neural control in nematodes. Comments in Theoretical Biology, 3(2):109-139, 1993.

[58] E. Niebur and P. Erdös. Theory of the locomotion of nematodes: control of the somatic motor neurons by interneurons. Mathematical Biosciences, 118:51-82, 1993.

[59] David Owald, Johannes Felsenberg, Clifford B. Talbot, Gaurav Das, Emmanuel Perisse, Wolf Huetteroth, and Scott Waddell. Activity of Defined Mushroom Body Output Neurons Underlies Learned Olfactory Behavior in Drosophila. Neuron, 86(2):417-427, April 2015.

[60] David Owald, Suewei Lin, and Scott Waddell. Light, heat, action: neural control of fruit fly behaviour. Philosophical Transactions of the Royal Society B: Biological Sciences, 370(1677):20140211, September 2015.

[61] David Owald and Scott Waddell. Olfactory learning skews mushroom body output pathways to steer behavioral choice in Drosophila. Current Opinion in Neurobiology, 35:178-184, December 2015.

[62] T.-P. Pai, C.-C. Chen, H.-H. Lin, A.-L. Chin, J. S.-Y. Lai, P.-T. Lee, T. Tully, and A.-S. Chiang. Drosophila ORB protein in two mushroom body output neurons is necessary for long-term memory formation. Proceedings of the National Academy of Sciences, 110(19):7898-7903, May 2013.

[63] A. Pascual. Localization of Long-Term Memory Within the Drosophila Mushroom Body. Science, 294(5544):1115-1117, November 2001.

[64] Emmanuel Perisse, David Owald, Oliver Barnstedt, Clifford B. Talbot, Wolf Huetteroth, and Scott Waddell. Aversive Learning and Appetitive Motivation Toggle Feed-Forward Inhibition in the Drosophila Mushroom Body. Neuron, 90(5):1086-1099, June 2016.

[65] Barret D. Pfeiffer, Teri-T B. Ngo, Karen L. Hibbard, Christine Murphy, Arnim Jenett, James W. Truman, and Gerald M. Rubin. Refinement of Tools for Targeted Gene Expression in Drosophila. Genetics, 186(2):735-755, October 2010.

[66] Pierre-Yves Plaçais, Séverine Trannoy, Anja B. Friedrich, Hiromu Tanimoto, and Thomas Preat. Two Pairs of Mushroom Body Efferent Neurons Are Required for Appetitive Long-Term Memory Retrieval in Drosophila. Cell Reports, 5(3):769-780, November 2013. 
bioRxiv preprint doi: https://doi.org/10.1101/2020.12.06.413500; this version posted March 4, 2021. The copyright holder for this preprint (which was not certified by peer review) is the author/funder. All rights reserved. No reuse allowed without permission.

[67] Christopher J Potter, Bosiljka Tasic, Emilie V Russler, Liang Liang, and Liqun Luo. The Q System: A Repressible Binary System for Transgene Expression, Lineage Tracing, and Mosaic Analysis. Cell, 141(3):536-548, April 2010.

[68] W. G. Quinn, W. A. Harris, and S. Benzer. Conditioned Behavior in Drosophila melanogaster. Proceedings of the National Academy of Sciences, 71(3):708-712, March 1974.

[69] Thomas A Ravenscroft, Jasper Janssens, Pei-Tseng Lee, Burak Tepe, Paul C Marcogliese, Samira Makhzami, Todd C Holmes, Stein Aerts, and Hugo J Bellen. Drosophila voltage-gated sodium channels are only expressed in active neurons and are localized to distal axonal initial segment-like domains. Journal of Neuroscience, 40(42):7999-8024, 2020.

[70] Clifton C Rumsey and Larry F Abbott. Synaptic democracy in active dendrites. Journal of Neurophysiology, 96(5):2307-2318, 2006.

[71] Bert Sakmann, editor. Single-Channel Recording. Springer, New York [u.a], 2. ed edition, 2009.

[72] Louis K Scheffer, C Shan Xu, Michal Januszewski, Zhiyuan Lu, Shin-ya Takemura, Kenneth J Hayworth, Gary B Huang, Kazunori Shinomiya, Jeremy Maitlin-Shepard, Stuart Berg, Jody Clements, Philip M Hubbard, William T Katz, Lowell Umayam, Ting Zhao, David Ackerman, Tim Blakely, John Bogovic, Tom Dolafi, Dagmar Kainmueller, Takashi Kawase, Khaled A Khairy, Laramie Leavitt, Peter H Li, Larry Lindsey, Nicole Neubarth, Donald J Olbris, Hideo Otsuna, Eric T Trautman, Masayoshi Ito, Alexander S Bates, Jens Goldammer, Tanya Wolff, Robert Svirskas, Philipp Schlegel, Erika Neace, Christopher J Knecht, Chelsea X Alvarado, Dennis A Bailey, Samantha Ballinger, Jolanta A Borycz, Brandon S Canino, Natasha Cheatham, Michael Cook, Marisa Dreher, Octave Duclos, Bryon Eubanks, Kelli Fairbanks, Samantha Finley, Nora Forknall, Audrey Francis, Gary Patrick Hopkins, Emily M Joyce, SungJin Kim, Nicole A Kirk, Julie Kovalyak, Shirley A Lauchie, Alanna Lohff, Charli Maldonado, Emily A Manley, Sari McLin, Caroline Mooney, Miatta Ndama, Omotara Ogundeyi, Nneoma Okeoma, Christopher Ordish, Nicholas Padilla, Christopher M Patrick, Tyler Paterson, Elliott E Phillips, Emily M Phillips, Neha Rampally, Caitlin Ribeiro, Madelaine K Robertson, Jon Thomson Rymer, Sean M Ryan, Megan Sammons, Anne K Scott, Ashley L Scott, Aya Shinomiya, Claire Smith, Kelsey Smith, Natalie L Smith, Margaret A Sobeski, Alia Suleiman, Jackie Swift, Satoko Takemura, Iris Talebi, Dorota Tarnogorska, Emily Tenshaw, Temour Tokhi, John J Walsh, Tansy Yang, Jane Anne Horne, Feng Li, Ruchi Parekh, Patricia K Rivlin, Vivek Jayaraman, Marta Costa, Gregory SXE Jefferis, Kei Ito, Stephan Saalfeld, Reed George, Ian A Meinertzhagen, Gerald M Rubin, Harald F Hess, Viren Jain, and Stephen M Plaza. A connectome and analysis of the adult Drosophila central brain. eLife, 9:e57443, September 2020.

[73] Johannes Schindelin, Ignacio Arganda-Carreras, Erwin Frise, Verena Kaynig, Mark Longair, Tobias Pietzsch, Stephan Preibisch, Curtis Rueden, Stephan Saalfeld, Benjamin Schmid, Jean-Yves Tinevez, Daniel James White, Volker Hartenstein, Kevin Eliceiri, Pavel Tomancak, and Albert Cardona. Fiji: An open-source platform for biological-image analysis. Nature Methods, 9(7):676-682, July 2012.

[74] Julien Séjourné, Pierre-Yves Plaçais, Yoshinori Aso, Igor Siwanowicz, Séverine Trannoy, Vladimiros Thoma, Stevanus R Tedjakumala, Gerald M Rubin, Paul Tchénio, Kei Ito, Guillaume Isabel, Hiromu Tanimoto, and Thomas Preat. Mushroom body efferent neurons responsible for aversive olfactory memory retrieval in Drosophila. Nature Neuroscience, 14(7):903-910, July 2011.

[75] Dominique Siegenthaler, Benjamin Escribano, Vanessa Bräuler, and Jan Pielage. Selective suppression and recall of long-term memories in Drosophila. PLoS biology, 17(8):e3000400, August 2019.

[76] Dominique Siegenthaler, Benjamin Escribano, Vanessa Bräuler, and Jan Pielage. Selective suppression and recall of long-term memories in Drosophila. PLoS Biology, 17(8):e3000400, August 2019.

[77] David C Sterratt, Martine R Groen, Rhiannon M Meredith, and Arjen Van Ooyen. Spine calcium transients induced by synaptically-evoked action potentials can predict synapse location and establish synaptic democracy. PLoS Comput Biol, 8(6):e1002545, 2012.

[78] Hailing Su and Diane K O’Dowd. Fast synaptic currents in drosophila mushroom body kenyon cells are mediated by $\alpha$-bungarotoxin-sensitive nicotinic acetylcholine receptors and picrotoxin-sensitive gaba receptors. Journal of Neuroscience, 23(27):9246-9253, 2003.

[79] Sean T Sweeney, Kendal Broadie, John Keane, Heiner Niemann, and Cahir J O'Kane. Targeted expression of tetanus toxin light chain in Drosophila specifically eliminates synaptic transmission and causes behavioral defects. Neuron, 14(2):341-351, February 1995. 
[80] Shin-Ya Takemura, Yoshinori Aso, Toshihide Hige, Allan Wong, Zhiyuan Lu, C Shan Xu, Patricia K Rivlin, Harald Hess, Ting Zhao, Toufiq Parag, Stuart Berg, Gary Huang, William Katz, Donald J Olbris, Stephen Plaza, Lowell Umayam, Roxanne Aniceto, Lei-Ann Chang, Shirley Lauchie, Omotara Ogundeyi, Christopher Ordish, Aya Shinomiya, Christopher Sigmund, Satoko Takemura, Julie Tran, Glenn C Turner, Gerald M Rubin, and Louis K Scheffer. A connectome of a learning and memory center in the adult Drosophila brain. eLife, 6:5643, July 2017.

[81] Shin-ya Takemura, Yoshinori Aso, Toshihide Hige, Allan Wong, Zhiyuan Lu, C Shan Xu, Patricia K Rivlin, Harald Hess, Ting Zhao, Toufiq Parag, Stuart Berg, Gary Huang, William Katz, Donald J Olbris, Stephen Plaza, Lowell Umayam, Roxanne Aniceto, Lei-Ann Chang, Shirley Lauchie, Omotara Ogundeyi, Christopher Ordish, Aya Shinomiya, Christopher Sigmund, Satoko Takemura, Julie Tran, Glenn C Turner, Gerald M Rubin, and Louis K Scheffer. A connectome of a learning and memory center in the adult Drosophila brain. eLife, 6:e26975, July 2017.

[82] Shin-ya Takemura, Yoshinori Aso, Toshihide Hige, Allan Wong, Zhiyuan Lu, C Shan Xu, Patricia K Rivlin, Harald Hess, Ting Zhao, Toufiq Parag, et al. A connectome of a learning and memory center in the adult Drosophila brain. Elife, 6:e26975, 2017.

[83] Nobuaki K Tanaka, Hiromu Tanimoto, and KEI Ito. Neuronal assemblies of the drosophila mushroom body. Journal of Comparative Neurology, 508(5):711-755, 2008.

[84] Lin Tian, S Andrew Hires, Tianyi Mao, Daniel Huber, M Eugenia Chiappe, Sreekanth H Chalasani, Leopoldo Petreanu, Jasper Akerboom, Sean A McKinney, Eric R Schreiter, Cornelia I Bargmann, Vivek Jayaraman, Karel Svoboda, and Loren L Looger. Imaging neural activity in worms, flies and mice with improved GCaMP calcium indicators. Nature Methods, 6(12):875-881, November 2009.

[85] Seth M. Tomchik and Ronald L. Davis. Dynamics of Learning-Related cAMP Signaling and Stimulus Integration in the Drosophila Olfactory Pathway. Neuron, 64(4):510-521, November 2009.

[86] Tim Tully and William G. Quinn. Classical conditioning and retention in normal and mutantDrosophila melanogaster. Journal of Comparative Physiology A, 157(2):263-277, March 1985.

[87] Glenn C. Turner, Maxim Bazhenov, and Gilles Laurent. Olfactory Representations by Drosophila Mushroom Body Neurons. Journal of Neurophysiology, 99(2):734-746, February 2008.

[88] Katrin Vogt, Christopher Schnaitmann, Kristina V Dylla, Stephan Knapek, Yoshinori Aso, Gerald M Rubin, and Hiromu Tanimoto. Shared mushroom body circuits underlie visual and olfactory memories in Drosophila. eLife, 3:e02395, August 2014.

[89] Leslie B Vosshall. Olfaction in Drosophila. Current Opinion in Neurobiology, 10(4):498-503, August 2000.

[90] Leslie B Vosshall, Hubert Amrein, Pavel S Morozov, Andrey Rzhetsky, and Richard Axel. A Spatial Map of Olfactory Receptor Expression in the Drosophila Antenna. Cell, 96(5):725-736, March 1999.

[91] Carmina Carelia Warth Pérez Arias, Patrizia Frosch, André Fiala, and Thomas D. Riemensperger. Stochastic and Arbitrarily Generated Input Patterns to the Mushroom Bodies Can Serve as Conditioned Stimuli in Drosophila. Frontiers in Physiology, 11:53, February 2020.

[92] R. I. Wilson. Transformation of Olfactory Representations in the Drosophila Antennal Lobe. Science, 303(5656):366-370, January 2004.

[93] Jie-Kai Wu, Chu-Yi Tai, Kuan-Lin Feng, Shiu-Ling Chen, Chun-Chao Chen, and Ann-Shyn Chiang. Long-term memory requires sequential protein synthesis in three subsets of mushroom body output neurons in Drosophila. Scientific Reports, 7(1):7112, December 2017.

[94] Nobuhiro Yamagata, Toshiharu Ichinose, Yoshinori Aso, Pierre-Yves Plaçais, Anja B. Friedrich, Richard J. Sima, Thomas Preat, Gerald M. Rubin, and Hiromu Tanimoto. Distinct dopamine neurons mediate reward signals for short- and long-term memories. Proceedings of the National Academy of Sciences, 112(2):578-583, January 2015.

[95] Zhihao Zheng, J Scott Lauritzen, Eric Perlman, Camenzind G Robinson, Matthew Nichols, Daniel Milkie, Omar Torrens, John Price, Corey B Fisher, Nadiya Sharifi, Steven A Calle-Schuler, Lucia Kmecova, Iqbal J Ali, Bill Karsh, Eric T Trautman, John A Bogovic, Philipp Hanslovsky, Gregory S X E Jefferis, Michael Kazhdan, Khaled Khairy, Stephan Saalfeld, Richard D Fetter, and Davi D Bock. A Complete Electron Microscopy Volume of the Brain of Adult Drosophila melanogaster. Cell, 174(3):730-743.e22, July 2018. 
bioRxiv preprint doi: https://doi.org/10.1101/2020.12.06.413500; this version posted March 4, 2021. The copyright holder for this preprint (which was not certified by peer review) is the author/funder. All rights reserved. No reuse allowed without permission.

\section{Supplementary Material}

\section{S1 Details of Electrophysiology}

\begin{tabular}{|c|c|c|c|c|c|}
\hline Sample & $V m[\mathrm{mV}]$ & $\tau_{m}[\mathrm{~ms}]$ & $C_{m}[\mathrm{pF}]$ & $C_{\text {spec }}\left[\mu \mathrm{F} / \mathrm{cm}^{2}\right]$ & $C_{\text {pass }}\left[\mathrm{S} / \mathrm{cm}^{2}\right]$ \\
\hline Cell 1 & -52.8 & 15.5 & 15.83 & 0.257 & 0.0000166 \\
Cell 2 & -63.1 & 12.2 & 13.21 & 0.214 & 0.0000176 \\
Cell 3 & -52.3 & 15.9 & 20.79 & 0.337 & 0.0000212 \\
Cell 4 & -59.2 & 10.7 & 12.35 & 0.200 & 0.0000187 \\
Cell 5 & -56.2 & 23.7 & 21.60 & 0.350 & 0.0000148 \\
\hline \hline Mean & -56.7 & 15.6 & 16.76 & 0.272 & 0.0000178 \\
SD & 4.5 & 5.0 & 4.26 & 0.069 & 0.0000024 \\
SEM & 2.0 & 2.3 & 1.90 & 0.031 & 0.0000011 \\
\hline
\end{tabular}

Table S1: Passive membrane properties of MBON- $\alpha 3$ calculated from electrophysiological measurements.

A

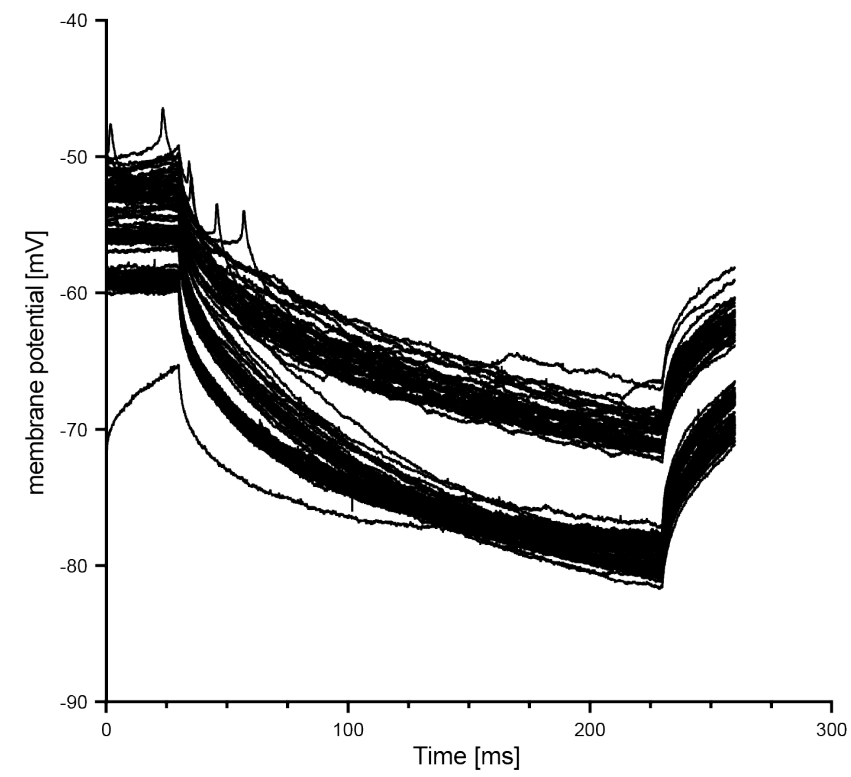

B

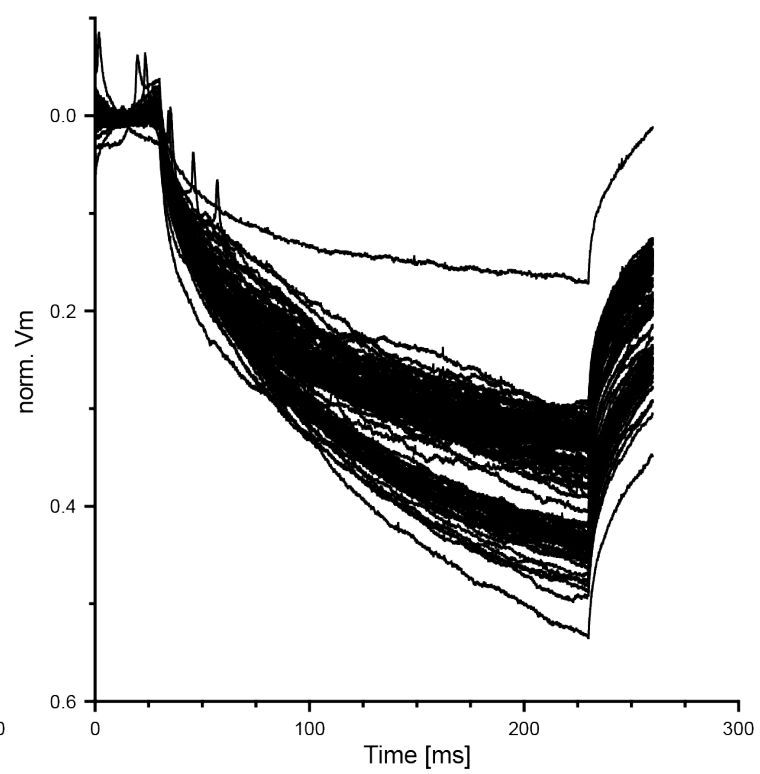

Figure S1: Raw traces from electrophysiological recordings of MBON- $\alpha 3$. (A) Raw traces of the induced membrane polarization after $200 \mathrm{~ms}$ current injections of $-10 p \mathrm{~A}$. We combined 35 trials of 3 different cells for the characterisation of the membrane time constant $\tau_{m}$ (B) Normalised data. 
A

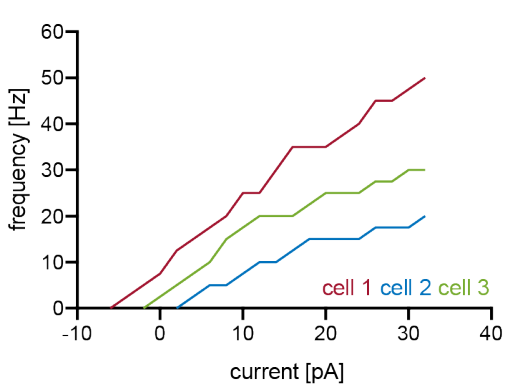

D

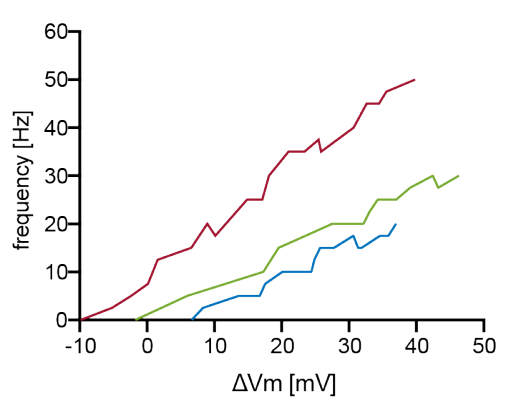

B

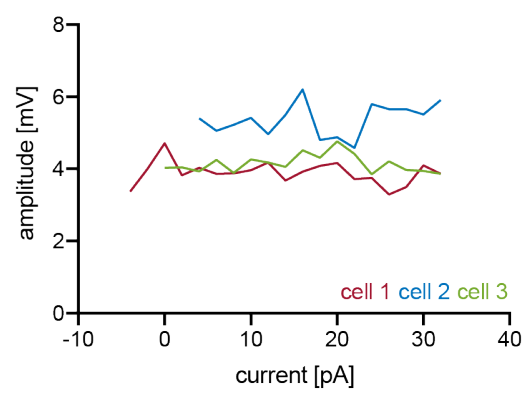

$\mathbf{E}$

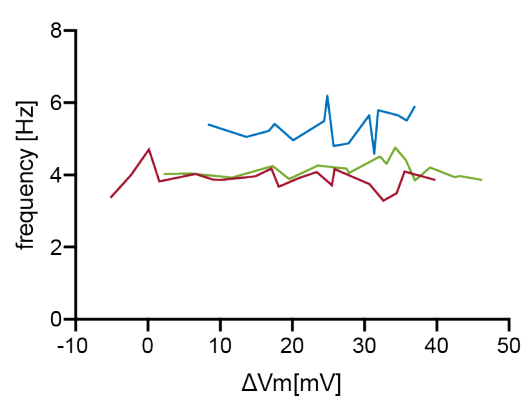

C

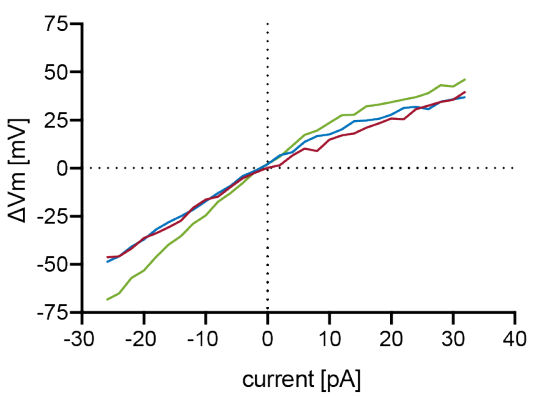

Figure S2: Effects of changes in membrane potential in MBON- $\alpha 3$. (A) Correlation between frequency of action potential firing and injected current. The frequency describes action potentials per second. (B) Relative change of spike amplitude after current injections. Spike amplitude corresponds to the peak of the highest action potential minus baseline. (C) Relative change of membrane potential after current injection. $\Delta \mathrm{Vm}$ is equal to the maximum depolarisation minus the resting membrane potential. (D)Relative change of spike frequency vs. $\Delta \mathrm{Vm}$ after current injection. (E) Relative change of spike amplitude vs. $\Delta$ Vm after current injections. 


\section{S2 Details of Model Implementation}

\section{S2.1 Morphological Parameters}

\begin{tabular}{|l|l|}
\hline Parameter & Value \\
\hline \hline number of sections & 4342 \\
\hline average section length & $1.24 \mu \mathrm{m}$ \\
\hline total length & $5377.39 \mu \mathrm{m}$ \\
\hline average diameter & $0.29 \mu \mathrm{m}$ \\
\hline average segment surface area & $1.26 \mu \mathrm{m}^{2}$ \\
\hline total surface area & $6168.32 \mu \mathrm{m}^{2}$ \\
\hline soma diameter & $6.45 \mu \mathrm{m}$ \\
\hline
\end{tabular}

Table S2: Whole model morphological parameters

A

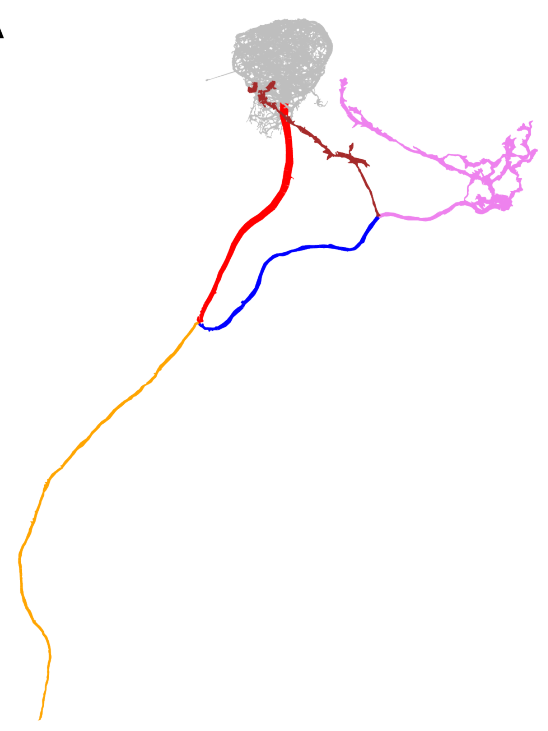

B

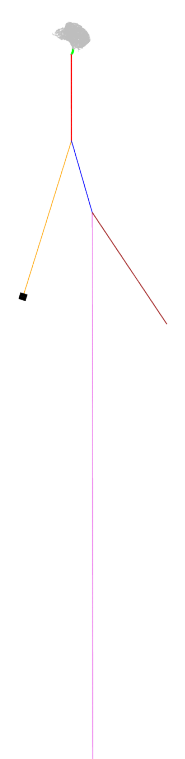

\begin{tabular}{|l|l|l|l|}
\hline Section & Length $[\mu \mathrm{m}]$ & Diameter $[\mu \mathrm{m}]$ & Number of Segments \\
\hline \hline Neurite 1 (red) & 75.89 & 1.14 & 27 \\
\hline Neurite 2 (orange) & 151.58 & 0.63 & 39 \\
\hline Branch 1 (blue) & 65.98 & 0.67 & 17 \\
\hline Branch 2 (violet) & 480.32 & 0.80 & 363 \\
\hline Branch 3 (brown) & 129.02 & 0.79 & 97 \\
\hline Soma (black) & 6.45 & 6.45 & 1 \\
\hline
\end{tabular}

Figure S3: Overview of axonal section and soma in the in silico model. The pink and brown sections represent the presynaptic release sites of $\mathrm{MBON}-\alpha 3$. Color-coded electron-microscopy based reconstruction (A) and in silico model (B). The reconstruction of the hemibrain did not include the complete axonal area (yellow) and the soma. (B) illustrates the appended sections and the corresponding diameter and lengths. The soma diameter (black) and axon length (yellow) were estimated from confocal microscopy images and literature. The table shows each section of the model (colors as in (A), B)) including length, diameter and number of segments, the latter corresponds to NEURON's 'nseg' variable for the respective section. 
bioRxiv preprint doi: https://doi.org/10.1101/2020.12.06.413500; this version posted March $4,2021$. The copyright holder for this preprint (which was not certified by peer review) is the author/funder. All rights reserved. No reuse allowed without permission.

\section{S2.2 Physiological Parameters}

\begin{tabular}{|c|c|c|c|}
\hline \multicolumn{4}{|c|}{ Electrophysiological Properties } \\
\hline Variable & Description & Fitted & Literature \\
\hline$R_{a}(\mathrm{Ra})$ & $\begin{array}{ll}\text { Cytoplasm } & \text { resistivity } \\
{[\Omega \times \mathrm{cm}]} & \end{array}$ & 85.41 & 30-400 (source $[10])$ \\
\hline$C_{m}(\mathrm{~cm})$ & $\begin{array}{l}\text { Specific membrane capac- } \\
\text { itance }\left[\mu \mathrm{F} / \mathrm{cm}^{2}\right]\end{array}$ & 0.6961 & $0.6-2.6$ (source $[10]$ ) \\
\hline$g_{\text {pas }}$ (pas.g) & $\begin{array}{l}\text { Passive membrane con- } \\
\text { ductance }\left[\mathrm{S} / \mathrm{cm}^{2}\right]\end{array}$ & $9.399 * 10^{-6}$ & $3.8 * 10^{-5}-1.2 * 10^{-4}($ source $[17])$ \\
\hline$e_{\text {pas }}($ pas.e) & $\begin{array}{lll}\text { Leak } & \text { reversal potential } \\
{[\mathrm{mV}]} & & \\
\end{array}$ & -55.64 & $-60($ source $[8])$ \\
\hline \multicolumn{4}{|c|}{ Synaptic Parameters } \\
\hline$\tau_{s}(\mathrm{tau})$ & $\begin{array}{l}\text { time to max. conductance } \\
{[\mathrm{ms}]}\end{array}$ & N.A. & 0.44 (source $[78]$ ) \\
\hline $\mathrm{E}$ & $\begin{array}{l}\text { synaptic current reversal } \\
\text { potential }[\mathrm{mV}]\end{array}$ & N.A. & $8.9($ source $[78])$ \\
\hline$G_{\max }(\operatorname{gmax})$ & $\begin{array}{l}\text { maximal conductance } \\
{[\mu m h o]}\end{array}$ & N.A. & $1.5627 * 10^{-5}($ source $[37])$ \\
\hline
\end{tabular}

Table S3: Electrophysiological values applied for the in silico model of MBON- $\alpha 3$-A. Parameter values were obtained from the literature or based on the fitting to our electrophysiological data. The first column shows the parameter names used in the NEURON environment in parentheticals. The maximal conductance was determined to achieve the target MBON depolarization from monosynaptic KC innervation 
bioRxiv preprint doi: https://doi.org/10.1101/2020.12.06.413500; this version posted March 4, 2021. The copyright holder for this preprint (which was not certified by peer review) is the author/funder. All rights reserved. No reuse allowed without permission.

A

\begin{tabular}{|c|c|c|c|c|c|}
\hline 0 & 10 & $\frac{1}{20}$ & $\begin{array}{cc}30 & 40 \\
\text { distance to } \mathrm{PN} & {[\mu \mathrm{m}]}\end{array}$ & $\frac{1}{50}$ & $\frac{1}{60}$ \\
\hline
\end{tabular}

B

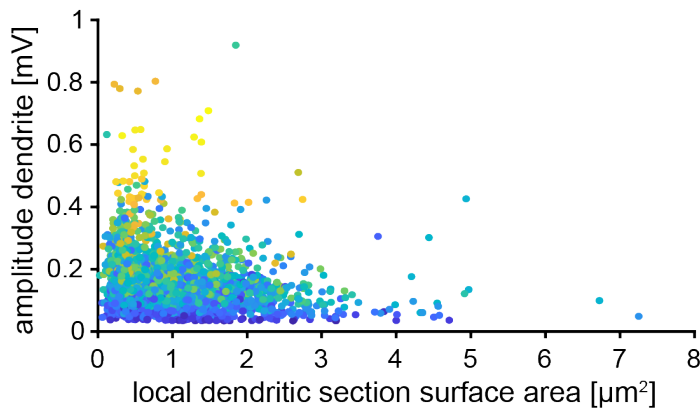

D

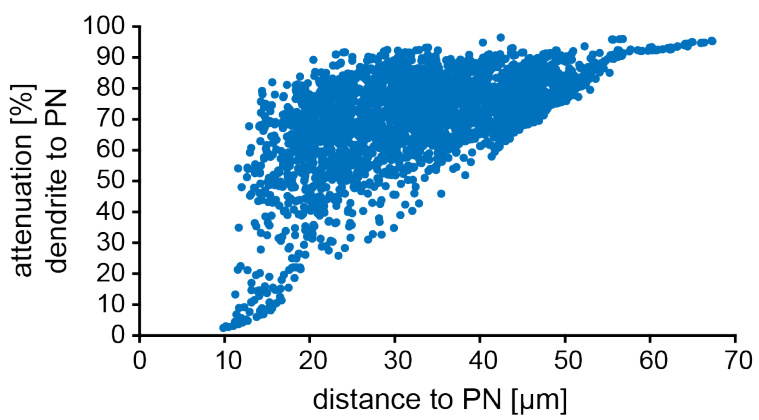

$\mathbf{F}$

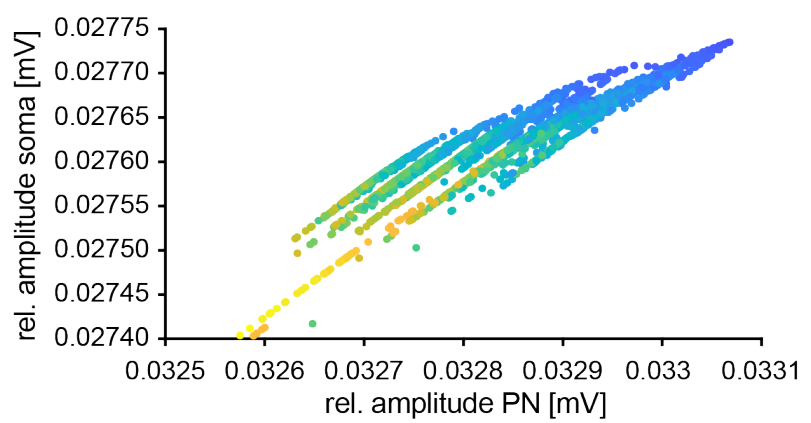

C

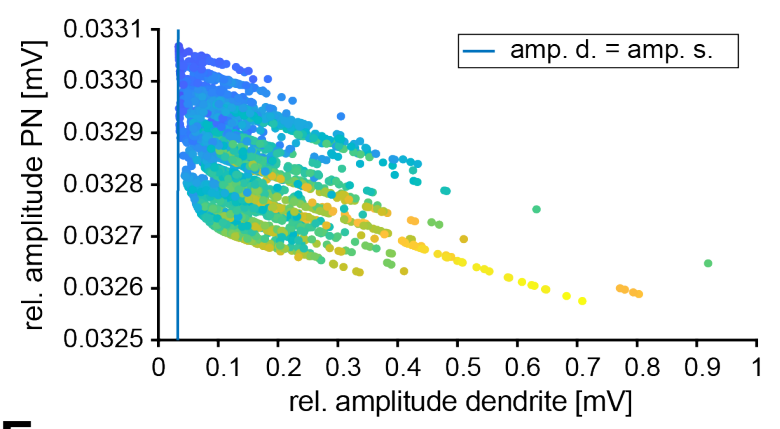

$\mathbf{E}$

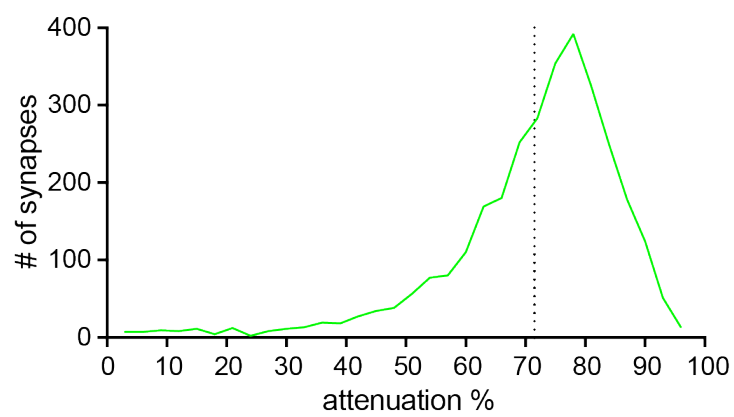

$\mathbf{G}$

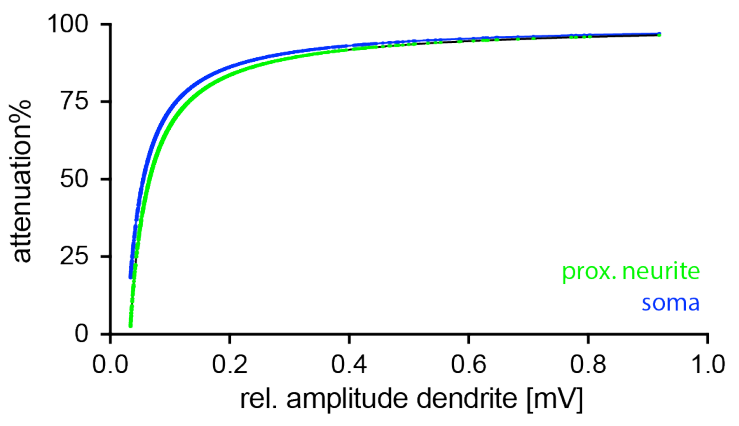

Figure S4: Voltage attenuation at the PN of MBON- $\alpha 3$. (A) Distance to the PN for each stimulated dendritic segment with the color code for the following graphs. (B) Scatter plot of the elicited amplitude in each stimulated dendritic section as function of its surface area. (C) Scatter plot of the voltage in the $\mathrm{PN}$ as function of the voltage in the dendrite, both relative to their baseline values. The blue line represents the line of identity. (D) Correlation of the measured distance to the PN with the percent of voltage attenuation in the soma for each synaptic section. (E) Distribution of the percentage of voltage attenuation in the soma per synapse. Vertical dotted line represents the mean attenuation in percent. (F) Scatter plot of the resulting amplitude at the PN plotted against the resulting amplitude at the soma, both relative to their baselines. $(\mathrm{G})$ Correlation of the attenuation in percent at the soma (blue) or at the proximal neurite (green) relative to the dendritic voltage. 
A

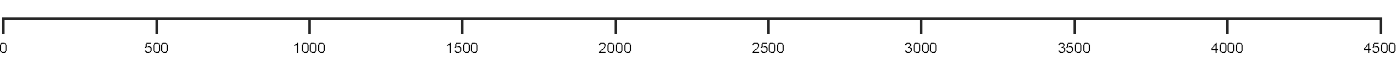

synapse sites

B

C
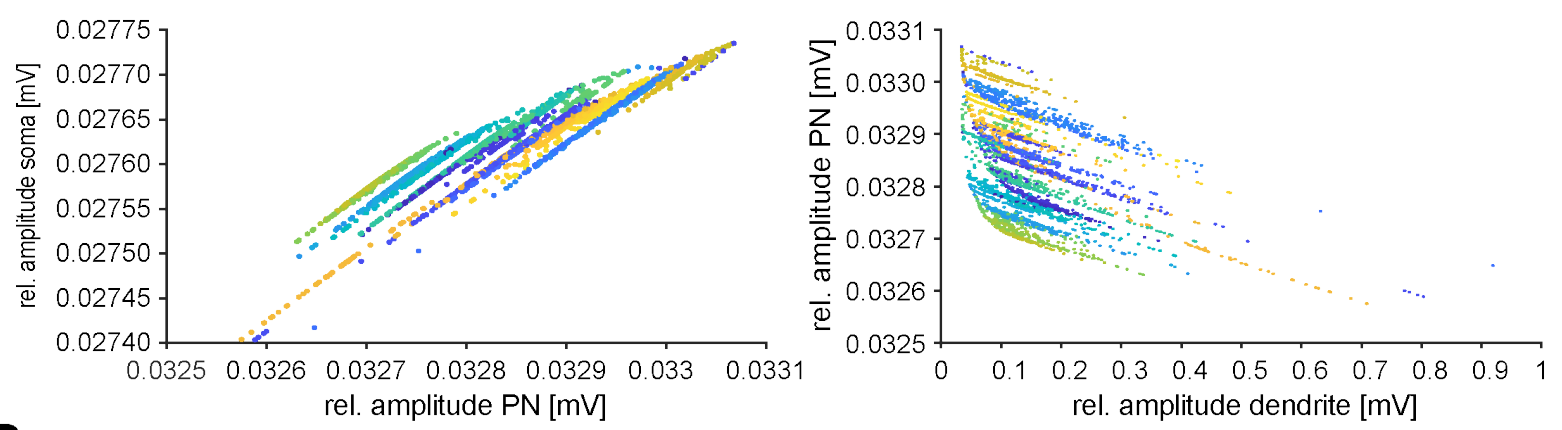

D

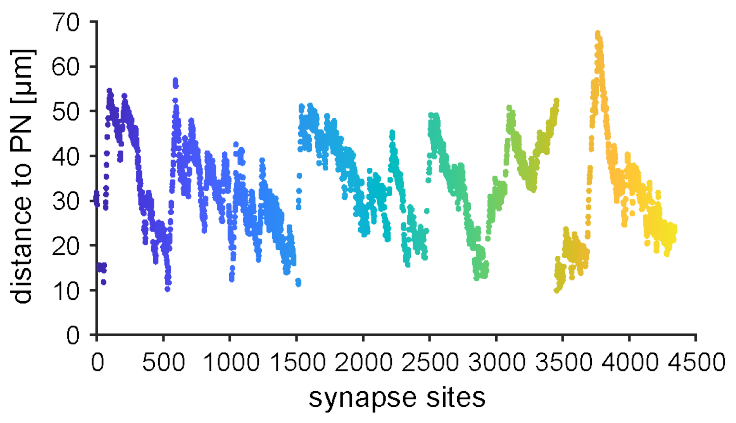

Figure S5: Branch-wise segment indexing and visualisation of branch-clusters in the data set. (A) Synaptic site index and corresponding color code for the following plots. (B) Scatter plot of the relative amplitude at the PN over the relative amplitude at the soma. (C) Scatter plot of relative amplitude at the PN over the relative amplitude at the dendrite. (D) Plot of the distance to the PN in $\mu \mathrm{m}$ for each synaptic site, sorted by index. 